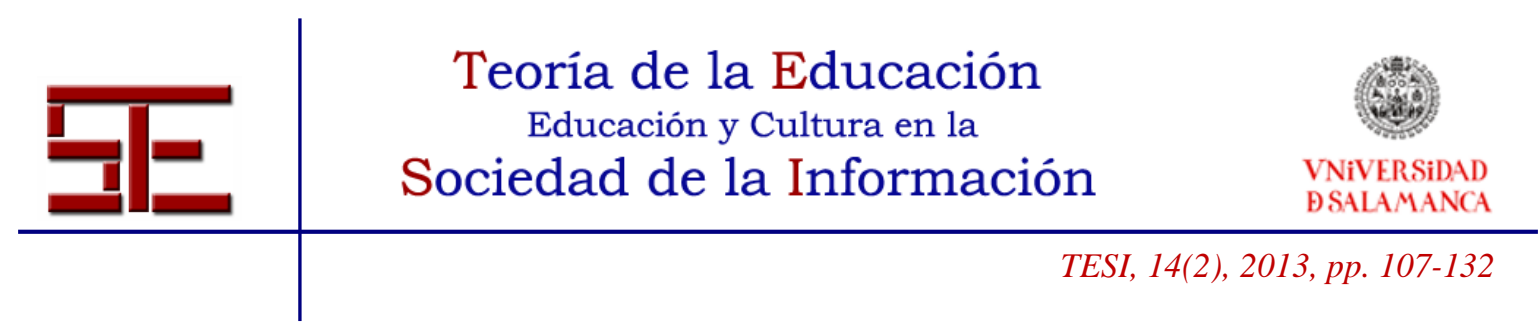

\title{
LOS MODELOS DE CONOCIMIENTO COMO AGENTES DE APRENDIZAJE SIGNIFICATIVO Y DE CREACIÓN DE CONOCIMIENTO
}

Resumen: El cambio educativo que propugna el contexto actual exige un desplazamiento del modelo conductista-positivista desgraciadamente predominante, que favorece el aprendizaje memorístico-mecánico, caldo de cultivo ideal para la existencia y mantenimiento de los errores conceptuales; a otro cognitivo-constructivista que estimule el aprendizaje significativo para permitir al alumno construir y dominar el conocimiento, por tanto ser más creativo y crítico.

Aquí se presenta un modelo de conocimiento donde el alumno construye nuevo conocimiento como consecuencia de aprender significativamente. Los alumnos desempeñan un papel activo aprendiendo no solo acerca del producto, sino del proceso mismo (metaconocimiento). También mostramos cÓmo promover la actividad del profesor fundamentalmente para crear las condiciones que faciliten la transformación a cargo del alumno de información en conocimiento útil, sustantivo, que se integre en su estructura de conocimiento en la memoria a largo plazo.

Por último, se proporcionan elementos para medir lo que el alumno sabe y valorar cómo ha cambiado su estructura cognitiva en relación con antiguos conocimientos, esto es, evaluar el necesario cambio conceptual.

Palabras clave: Aprendizaje significativo: Agentes de conocimiento; Creación de Conocimiento; Mapas Conceptuales; Espacio Europeo de Educación Superior.

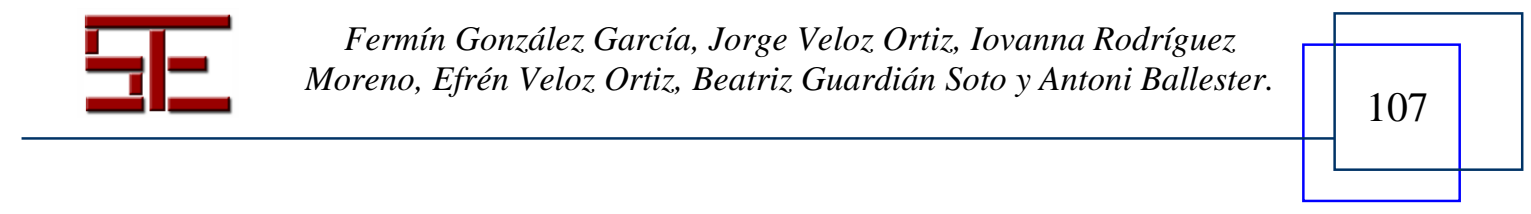




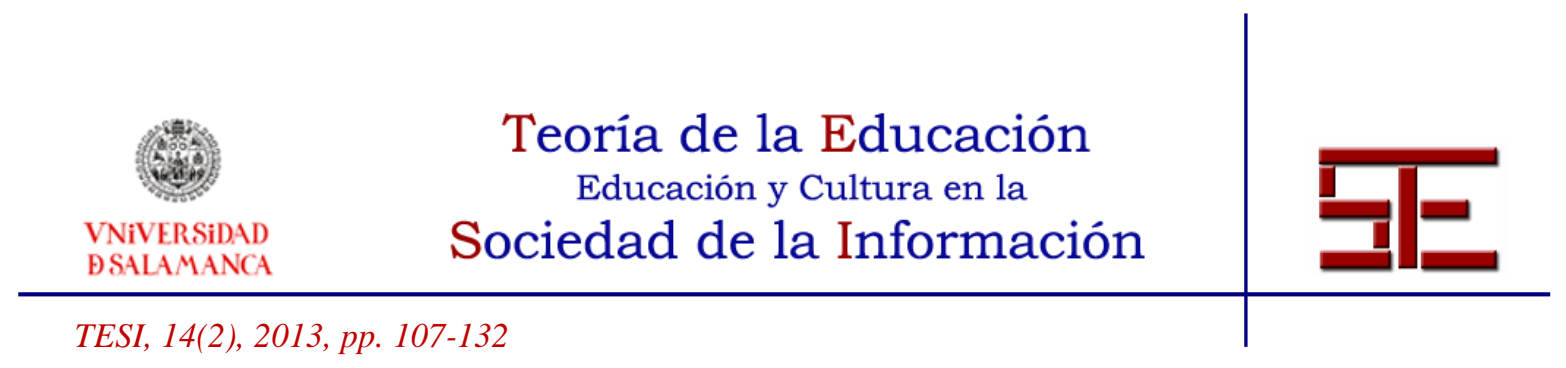

\title{
KNOWLEDGE MODELS AS AGENTS OF MEANINFUL LEARNING AND KNOWLEDGE CREATION
}

\begin{abstract}
The educational change that pushes the current context requires a shift in the unfortunately predominant positivist-behaviourist model that favours mechanical memoristic learning, ideal breeding ground for the existence and maintenance of conceptual errors, to another cognitive-constructivist that stimulates meaningful learning to allow students to build and master knowledge, therefore to be more creative and critical.
\end{abstract}

We present here a model of knowledge where students construct new knowledge as a result of significant learning. Students play an active role, learning not only about the product, but also about the process itself (meta-cognition). We also show how to promote teacher activity primarily in order to create the conditions that facilitate the student to transform the information in useful, substantive knowledge, to be incorporated in his knowledge structure and in his long-term memory.

Finally, we provide elements to measure what the student knows and to assess how their cognitive structure has changed regarding their ancient knowledge; that is, to assess the necessary conceptual change.

Key words: Meaningful learning; knowledge agents; Knowledge Creation; Concept Mapping; European Higher Education Area.

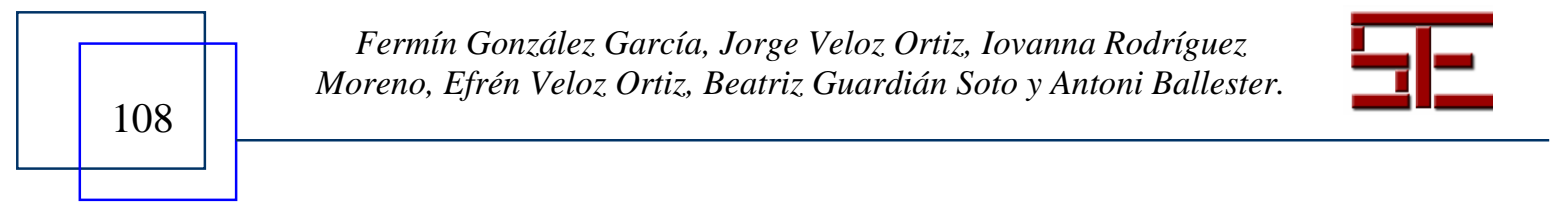




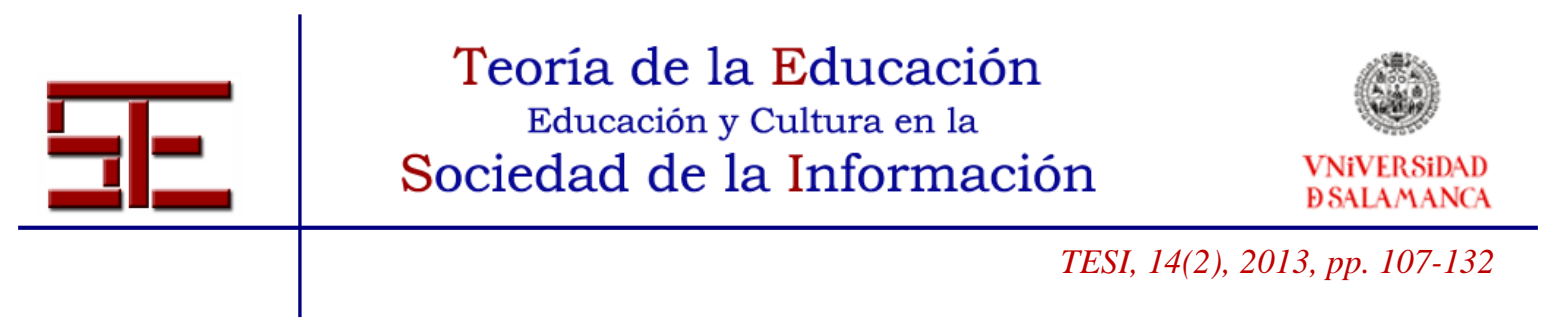

\section{LOS MODELOS DE CONOCIMIENTO COMO AGENTES DE APRENDIZAJE SIGNIFICATIVO Y DE CREACIÓN DE CONOCIMIENTO}

Fecha de recepción: 27/05/2013; fecha de aceptación: 20/06/2013; fecha de publicación: 30/07/2013

Fermín María González García.

fermin@unavarra.es

UPNA

Jorge Fernando Veloz Ortiz.

jveloz@ipn.mx

IPN

Iovanna Alejandra Rodríguez Moreno.

irodriguezm@ipn.mx

IPN

Luis Efrén Velos Ortiz.

leveloz@ipn.mx

IPN

Beatriz Guardián Soto

bggirasoles@yahoo.com.mx

IPN

Antoni Ballester Valori.

antoniballester@wanadoo.es

UIB

\section{1.- CONTEXTO ACTUAL}

Un análisis detallado del mapa conceptual bidimensional de la Figura 1 sobre la educación en el siglo XXI nos permite un discurso escrito lineal que transcribo en las líneas siguientes. En primer lugar nos presenta los retos a los que la educación del siglo XXI debe enfrentarse. Unos vienen de la llamada sociedad del conocimiento y de la información, que puede resumirse en un cambio de una ética de la obligación por otra de

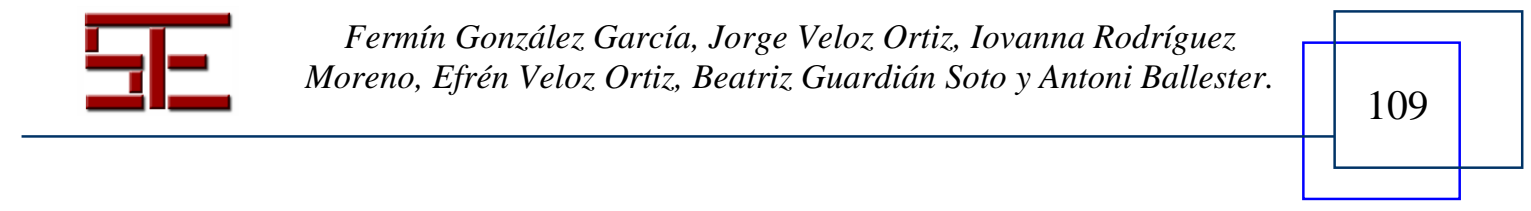




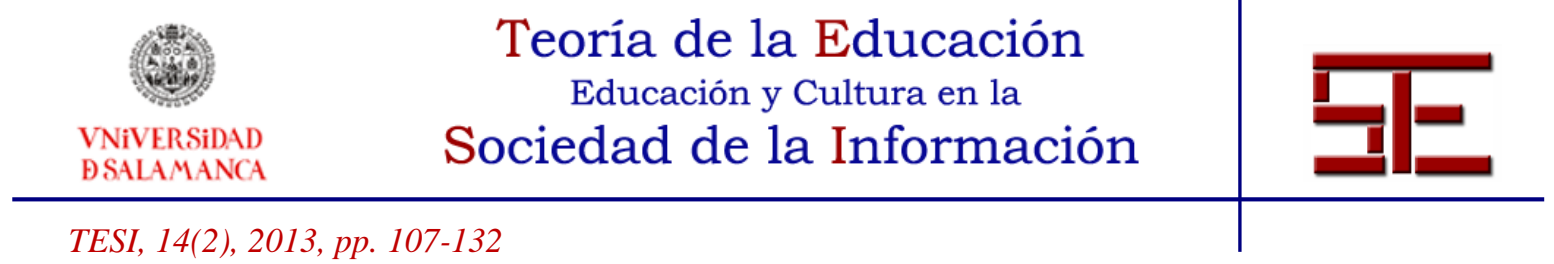

la responsabilidad, un uso generalizado de las tecnologías de la información y de la comunicación, las llamadas TIC, y la exigencia de una escuela, en términos genéricos, de calidad, con rendición de cuentas a la sociedad y que estimule en los alumnos/as, futuros trabajadores del conocimiento, una alfabetización universal, una motivación para aprender a aprender y una disciplina para un aprendizaje continuo. Otros provienen de la implantación del Espacio Europeo de Educación Superior (EEES) que comporta la exigencia de un cambio en el modelo de enseñanza/aprendizaje que hace hincapié en un nuevo rol no solo del docente, sino del alumno, lo que afecta, al nuevo diseño de las asignaturas recogido en las llamadas Guías Docentes (Zabalza, 2004).

El tercer reto se refiere al que plantean los modelos de gestión de la calidad. Identificada ésta como el grado de eficiencia y eficacia demostrada en la consecución de unos objetivos enmarcados en los conceptos Misión y Visión de la institución educativa, y naturalmente negociados y compartidos por la comunidad educativa en su totalidad. Pertinentes procesos de certificación y acreditación serán los garantes de que a través de una previa evaluación, con la consiguiente detección de puntos débiles y el adecuado planteamiento de estrategias de mejora, se haga operativa la mejora continua de la calidad.

El cambio educativo que propugna este contexto exige un desplazamiento del modelo conductista-positivista desgraciadamente predominante, que favorece el aprendizaje memorístico-mecánico, caldo de cultivo ideal para la existencia y mantenimiento de los errores conceptuales (González, Morón y Novak, 2001; Molina, Pozueta, González, in progress), a otro cognitivo-constructivista potenciador del aprendizaje significativo, que permita al alumno/a construir y dominar el conocimiento (Meichembaum; Biemiller, 1998) y ser, por tanto, más creativo y crítico. En este paradigma el alumno construye nuevo conocimiento como consecuencia de aprender significativamente. Los alumnos en este proceso desempeñan un papel activo aprendiendo no solo acerca del producto, sino del proceso mismo (metaconocimiento). La responsabilidad del profesor será fundamentalmente crear las condiciones que faciliten la transformación a cargo del alumno de información en conocimiento útil, sustantivo, que se integre en su estructura de conocimiento bien articulada, en la memoria a largo plazo.

La evaluación en este nuevo panorama educativo desempeña un papel clave como impulsora de la mejora continua, consolidando los puntos fuertes y corrigiendo los débiles. Tal evaluación deberá tener una doble dimensión (Novak, 1998): por un lado, medir lo que el alumno sabe y, por otro, valorar cómo ha cambiado su estructura cognitiva

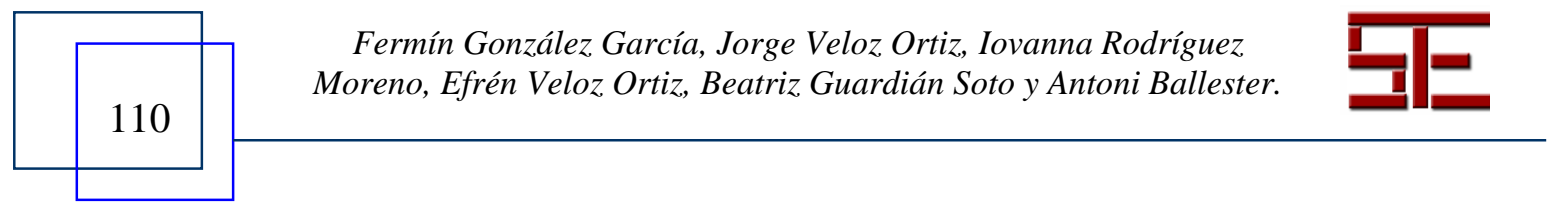




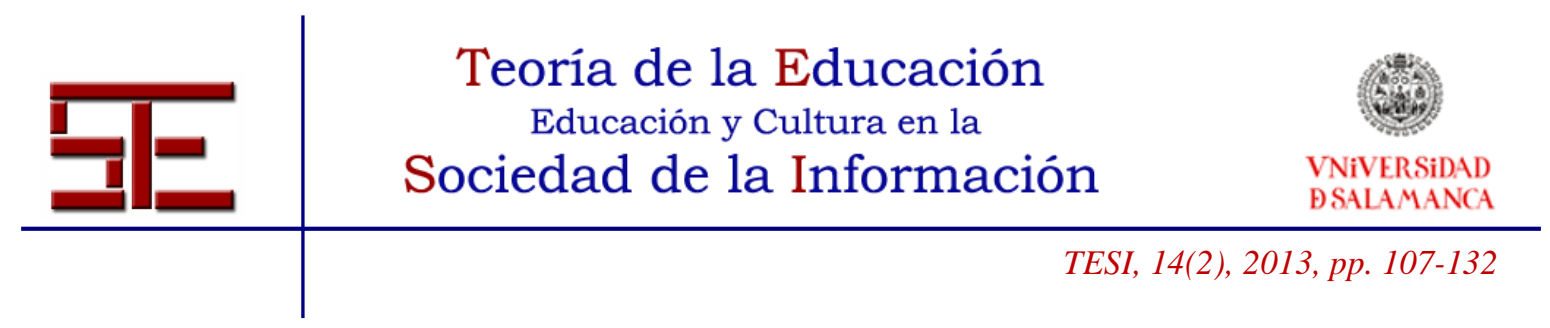

en relación con esos conocimientos, esto es, evaluar el necesario cambio conceptual (Posner, Strike, Hewson y Gertzog, 1982).

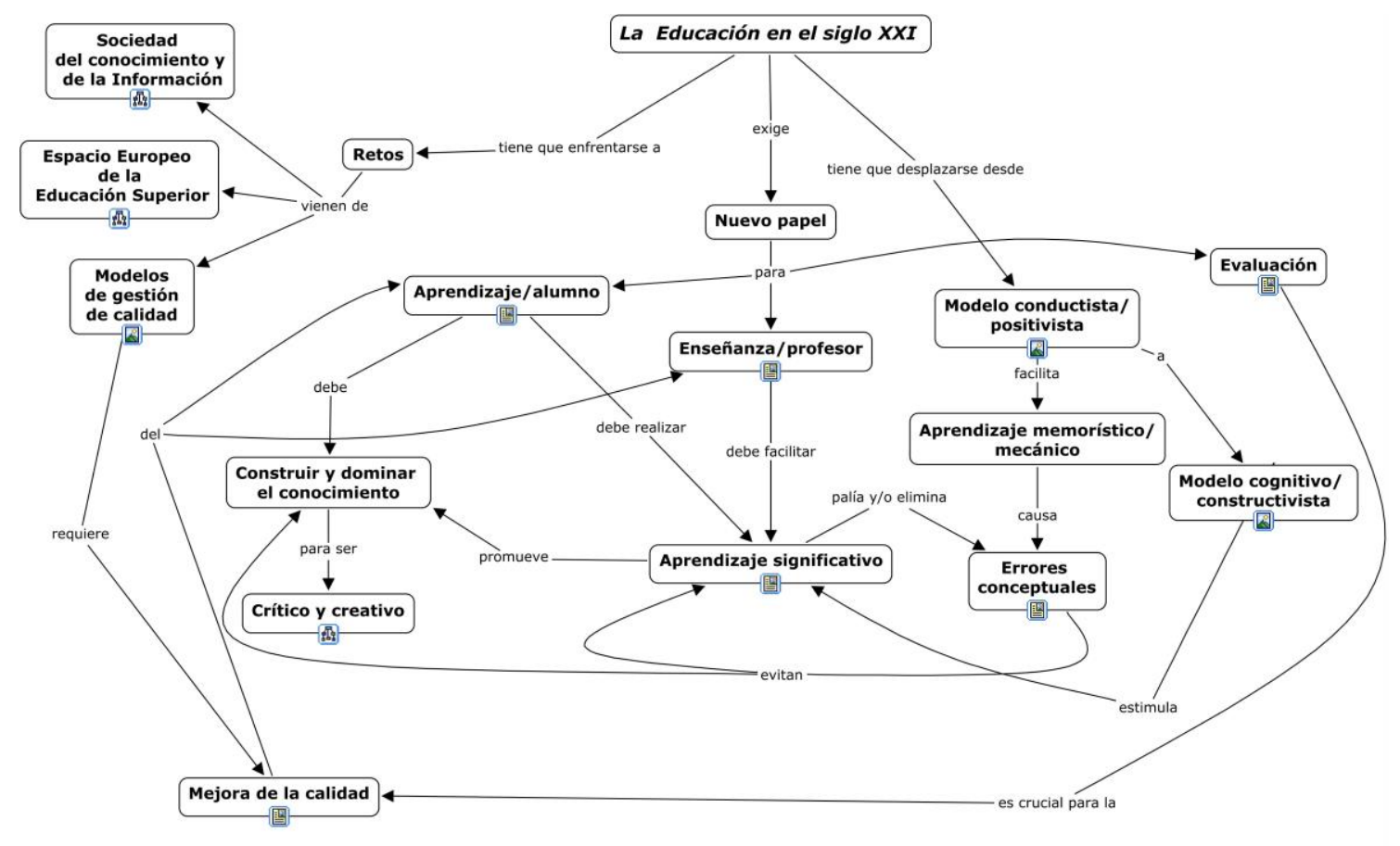

Figura 1. Mapa conceptual sobre la Educación en el siglo XXI (González, 2011)

La Declaración de Bolonia en 1999 sentó las bases para la construcción de un EEES, organizado conforme a ciertos principios (calidad, movilidad, diversidad, competitividad) y orientado hacia la consecución entre otros de dos objetivos estratégicos: el incremento del empleo en la Unión Europea y la conversión del Sistema Europeo de Formación Superior en un polo de atracción para estudiantes y profesores de otras partes del mundo.

Entre los objetivos la Declaración de Bolonia podemos citar: la adopción de un sistema fácilmente legible y comparable de titulaciones; la adopción de un sistema basado, fundamentalmente, en dos ciclos principales, grado y postgrado (máster y doctorado), y en el establecimiento de un sistema de créditos, como el sistema ECTS (Sistema europeo de transferencia y acumulación de créditos), y la promoción de la cooperación europea para asegurar un nivel de calidad para el desarrollo de criterios y metodologías

Fermín González García, Jorge Veloz Ortiz, Iovanna Rodríguez Moreno, Efrén Veloz Ortiz, Beatriz Guardián Soto y Antoni Ballester. 


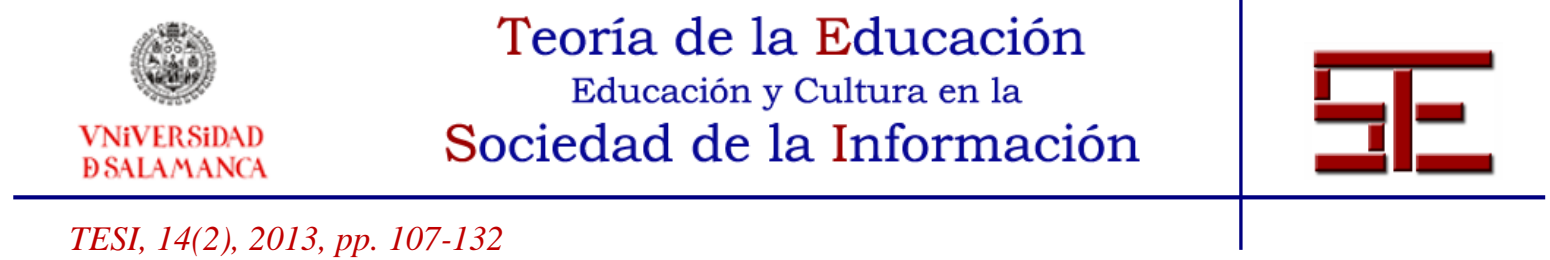

comparables. Son hasta la fecha 45 países los participantes en la construcción del EEES, que nace con vocación de liderazgo y de referente mundial en el ámbito de la educación superior.

En este nuevo contexto el rol clásico del profesor tiene que cambiar. Es un nuevo concepto el que está en la base de la educación donde la dimensión de la enseñanza (énfasis en el que enseña o en lo enseñado) está en función del que aprende y cómo aprenderá mejor y llegará a conseguir lo que se le ha marcado como objetivo. Es decir, es un concepto de educación basado en el aprendizaje y centrado en el alumno.

Es un concepto de enseñanza más amplio. Al profesor se le pide que guíe/acompañe al alumno a través de un conjunto de actividades educativas donde la clase presencial es un elemento para la consecución de una serie de competencias en las que los conocimientos (su comprensión y su manejo) son una parte. Según este concepto no son, naturalmente, sólo las horas de clase las que deben contarse como dedicación, sino el conjunto de las tareas que se le piden en cada caso, su planificación detallada, el seguimiento de las actividades o las experiencias educativas y su evaluación.

La calidad significa el grado de éxito que logre la educación superior para generar ambientes apropiados para la producción y transferencia de conocimientos y competencias genéricas y específicas a cada disciplina y a nuevos tipos de aprendizaje (González y Wagenaar, 2003).

Las universidades deben usar las potencialidades del proceso de Bolonia para promover la calidad en la enseñanza y el aprendizaje, definir resultados del aprendizaje adecuados y señalar la manera de lograrlos. De aquí que deban dedicar cuidadosa atención a sus enfoques de enseñanza y aprendizaje.

Además constituye un reto continuo para las universidades la mejora continua de la calidad docente, investigadora y de gestión. Las buenas prácticas en la Educación Superior suponen aumentar la competitividad de las universidades, asumir los compromisos del nuevo espacio universitario europeo y prestar un mejor servicio a la sociedad (Villar y Alegre, 2004).

En la Figura 2, se presenta un mapa conceptual ejemplo que hace visible este nuevo marco. Obsérvese en el mismo, la adecuación del marco teórico de Ausubel, Novak y Gowin, así como de los Mapas Conceptuales (MMCC) y del diagrama "V" de Gowin,

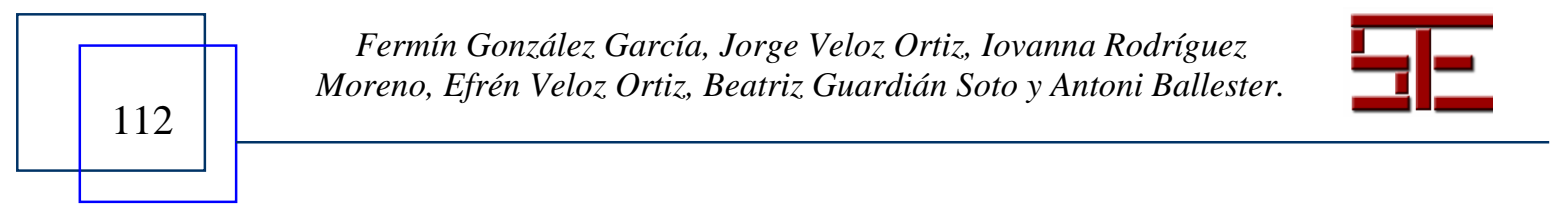




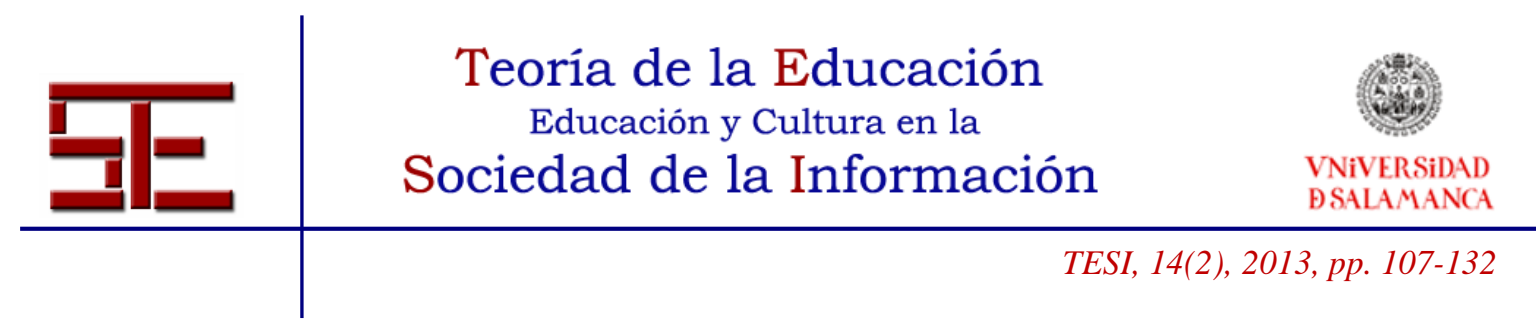

para acometer los cambios educativos que demanda el EEES. De una manera general los mapas conceptuales MMCC son diagramas que indican relaciones entre conceptos clave o entre palabras que usamos para representar conceptos y es una técnica propuesta y desarrollada por Joseph Novak (Novak y Gowin, 1988).

Los mapas conceptuales son instrumentos poderosos para describir estructuras de conocimiento disciplinar a través de jerarquías conceptuales o de significados, partiendo de reglas generales hacia las más específicas de manera clara y sin olvidar el sentido interpretativo y explicativo de quien lo produce o elabora.

Los iconos gráficos vinculados a conceptos del mapa conceptual pueden desplegarse haciendo clic sobre ellos y a continuación en la expresión escrita que aparece. Se visualiza así la información correspondiente. Al mapa original con los recursos asociados se puede acceder a través del programa informático de descarga gratuita CMapTools del Institute for Human and Machine Cognition (Cañas, 2004; Novak y Cañas, 2006), además a todos nuestros trabajos relacionados con docencia, gestión e investigación con utilización de MMCC se puede acceder libremente en el Servidor Público de la Universidad Pública de Navarra y otros más en la carpeta Fermín González.

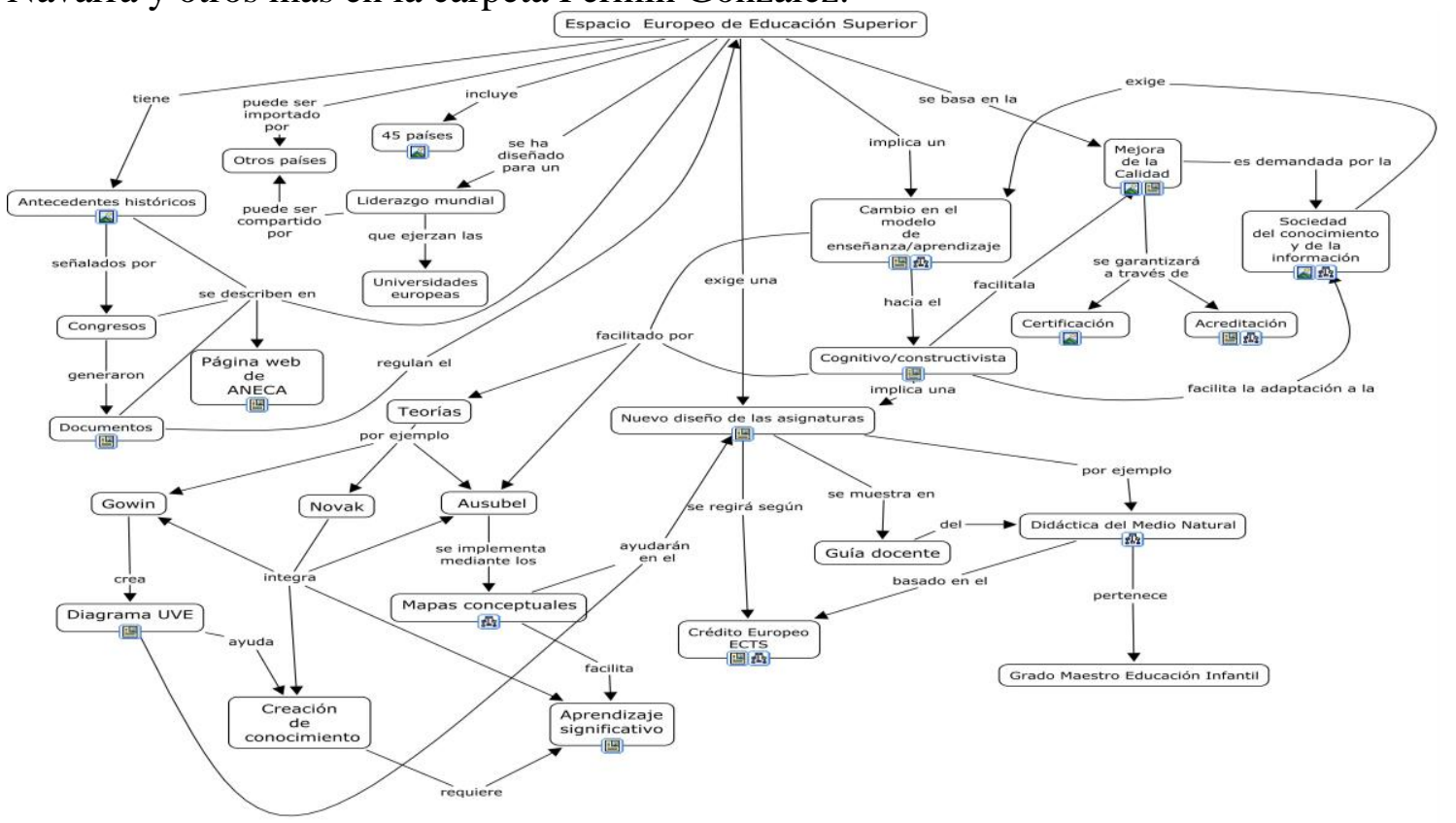

Figura 2. Mapa conceptual que representa el Espacio Europeo de Educación Superior y sus principales implicaciones educativas(González, 2011)

Fermín González García, Jorge Veloz Ortiz, Iovanna Rodríguez Moreno, Efrén Veloz Ortiz, Beatriz Guardián Soto y Antoni Ballester. 


Teoria de la Educación
Educación y Cultura en la
$\begin{gathered}\text { VNiVERSIDAD } \\ \text { BSALAMANCA }\end{gathered}$

Numerosos trabajos de investigación muestran evidencias que demuestran con creces el gran potencial que tienen los MMCC para la mejora de la docencia, tanto para la diagnosis de conocimientos previos de los alumnos/as como para el diseño e implementación consecuentes del currículum y la instrucción a cargo del profesor. Facilitándose así el gran reto del aprendizaje significativo y la construcción creativa de conocimientos por parte del alumno/a.

\section{2.- ELABORACIÓN DE MODELOS DE CONOCIMIENTO}

Nuestro equipo de investigación interdisciplinar en Didáctica de las Ciencias (IDIC) de la Universidad Pública de Navarra ha puesto en práctica una metodología basada en la elaboración de modelos de conocimiento (Cañas, 2004; González, Guruceaga, Pozueta y Lara, 2009; Zabalza, 2004). En el mapa conceptual de la Figura 3 se muestra la fundamentación teórica de esta práctica. Como se transparenta en el mapa se basa fundamentalmente en los marcos teóricos de Ausubel y Novak, que utiliza como herramientas operativas los MMCC y el diagrama "V". Los modelos de conocimiento construídos por los alumnos constituyen ejemplos de aprendizaje significativo y de construcción creativa de conocimientos (González, 2008; Ballester, 2002).

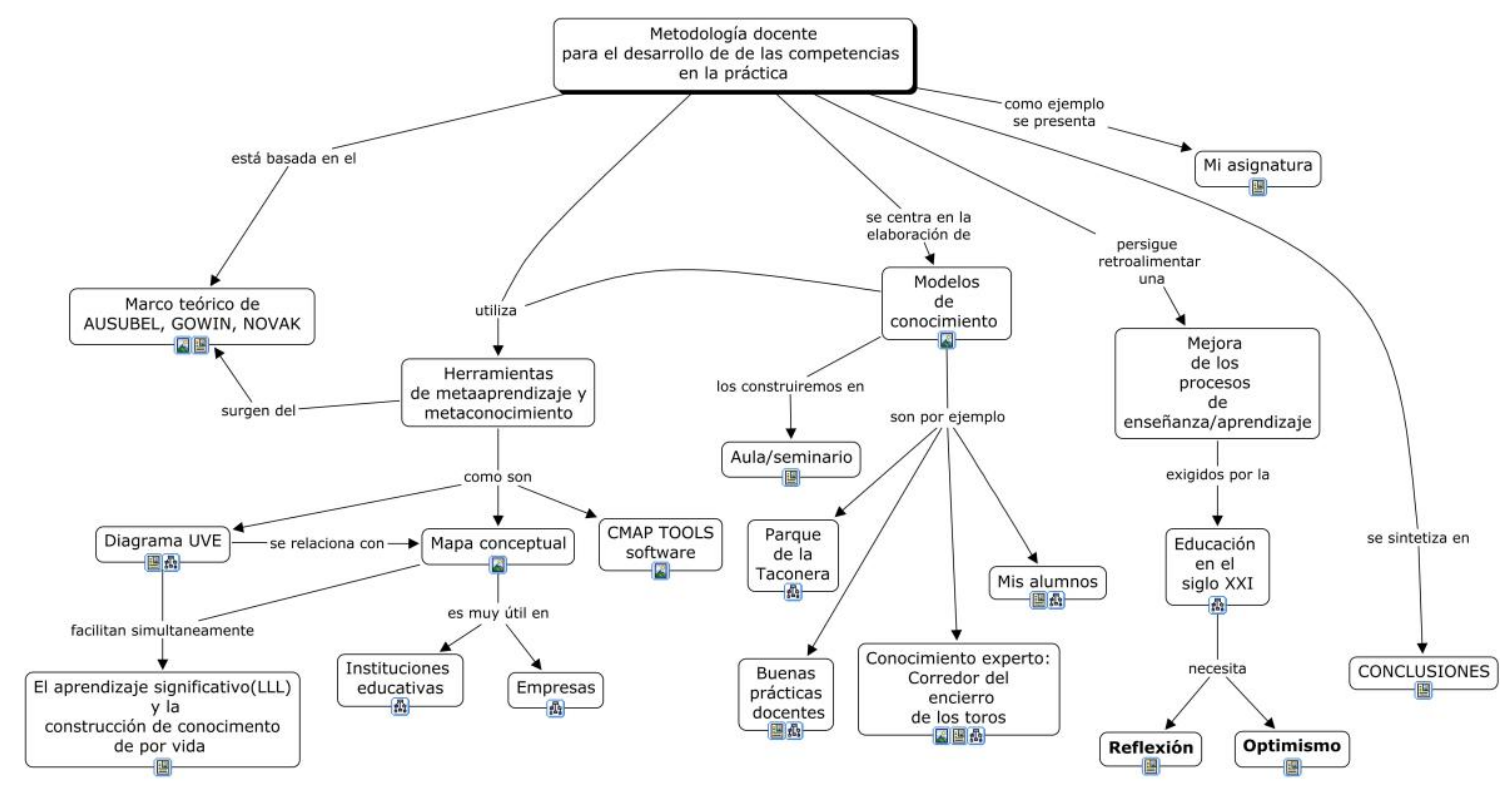

Figura 3. Mapa conceptual de una metodología docente basada en la construcción de modelos de conocimiento(González, 2011)

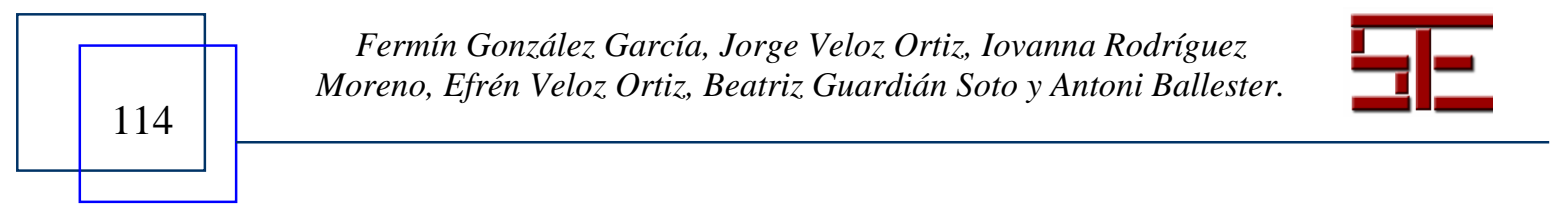




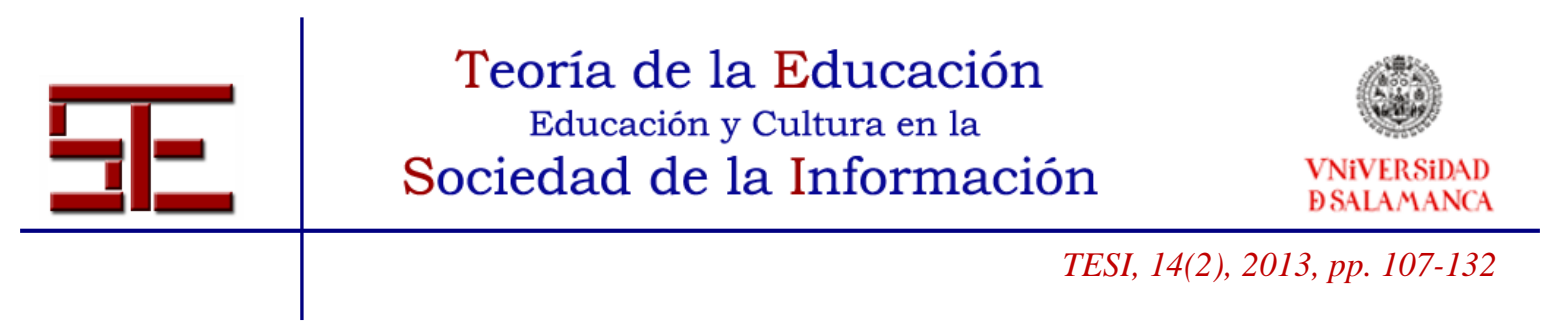

Un modelo de conocimiento está formado por un conjunto de MMCC y recursos digitalizados asociados a los mismos, y todo ello en relación con una temática determinada. En el caso que presentamos se trata del Parque de la Taconera de Pamplona. Como se describió en el Proyecto GONCA (Proyecto GONCA, 2003) nos propusimos la elaboración de un módulo de conocimiento del medio/entorno próximo y su edición en un CDRom, navegable por Internet y con seleccionados y contrastados recursos multimedia ligados a los diferentes nodos/conceptos de los MMCC. Pretendimos como uno de los objetivos elaborar modelos de conocimiento que facilitasen la construcción de aprendizajes significativos en nuestros alumnos, relacionados con aspectos botánicos, zoológicos y monumentales del Parque de la Taconera.

Con el fin de conseguir el objetivo citado nuestros alumnos elaboraron un modelo de conocimiento sobre el Parque de la Taconera de Pamplona utilizando técnicas instruccionales que contribuyeran a la mejora de los procesos de enseñanza-aprendizaje, como los MMCC. Para la elaboración de ese modelo nuestros alumnos recibieron una serie de materiales diseñados por los profesores de la Universidad así como el Programa informático CMapTools, que puede descargarse libremente y sin coste en la siguiente dirección electrónica en Internet: cmap.ihmc.us. Este programa creado en el Institute for Human and Machine Cognition (Cañas, 2004) permite a los alumnos construir, compartir y criticar conocimientos basados en MMCC.

Durante casi una de decena de años llevamos utilizando esta estrategia, con una amplia y rica experiencia y excelentes resultados, algunos de los cuales hemos presentado en diferentes jornadas y congresos y tesis doctorales (Guruceaga, 2001; Molina, Pozueta y González, in progress). Además este trabajo ha merecido el interés de colegas de otras partes del Estado español y de países en el extranjero. Es por ello que decidimos centrar la elaboración de modelos de conocimiento en esta temática del Parque de la Taconera.

Uno de los materiales entregados a los alumnos es un Mapa Conceptual sobre el Parque de la Taconera de Pamplona (ver Figura 4), que sirvió de punto de partida para la elaboración de módulos multimedia sobre temas de conocimiento del medio natural, social y cultural, no lineales y susceptibles de ser utilizados en los distintos niveles educativos. Este mapa figurará como trasfondo de todos los modelos de conocimiento que hemos elaborado sobre distintas temáticas del parque y que mostraremos en las figuras correspondientes. Es el gran foco que ilumina, da sentido y significado a los distintos conceptos, contextualizando toda la información aportada en los mapas.

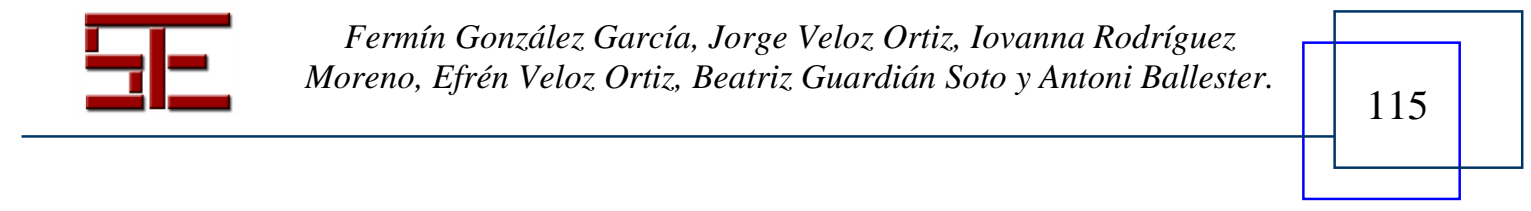




Teoria de la Educación
Educación y Cultura en la
$\begin{gathered}\text { VNiVERSIDAD } \\ \text { BSALAMANCA }\end{gathered}$

Este año hemos diseñado un nuevo proyecto que consiste en elaborar modelos de conocimiento por grupos de alumnos/as de las zonas en que está dividido el Campus de la Universidad Pública de Navarra. Así, integrándolos, conseguiremos construir un modelo de conocimiento global del campus que será navegable por Internet, a través del mapa conceptual como interfaz.

En el epígrafe correspondiente presentamos la asignatura en el marco de la cual se realiza la experiencia.

En el mapa conceptual de la Figura 4 se recoge información general del Parque de la Taconera, información referente no sólo a conceptos del Parque, sino también a actitudes y procedimientos. El parque lo consideramos como un ecosistema urbano que tiene un potencial educativo importante y que facilita la formación de hábitos de trabajo científico. Los conceptos de este mapa son lo suficientemente generales para subsumir la información correspondiente y orientan en la búsqueda eficiente y eficaz de la misma.

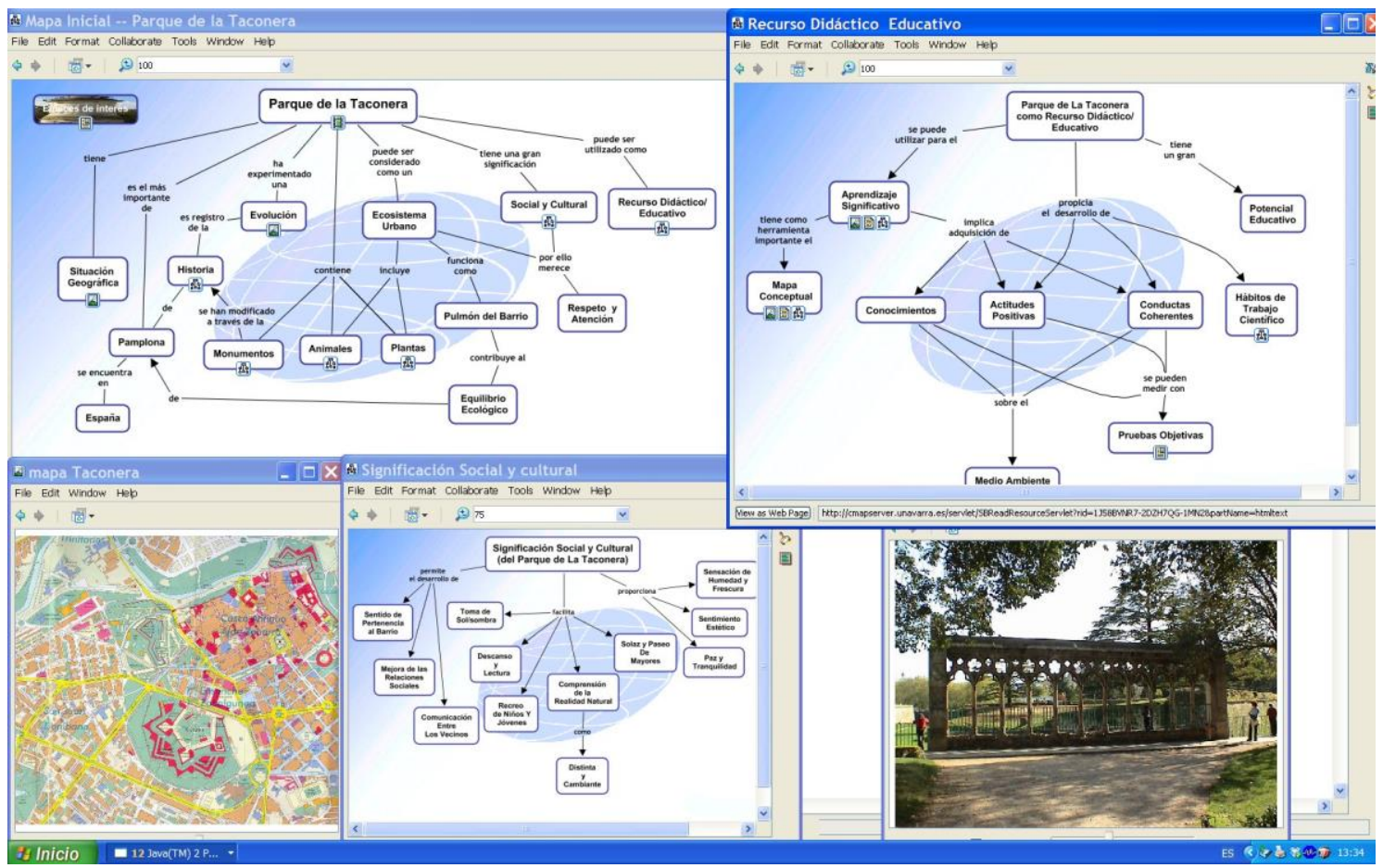

Figura 4. Mapa conceptual sobre el Parque de la Taconera, mostrando algunos recursos, vinculados a conceptos, abiertos.

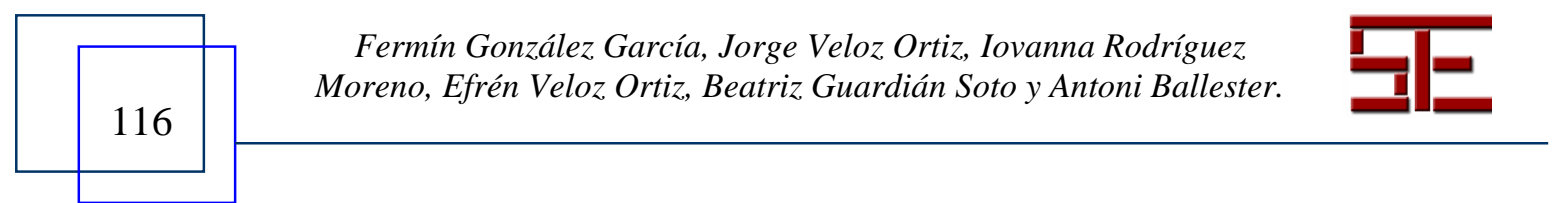




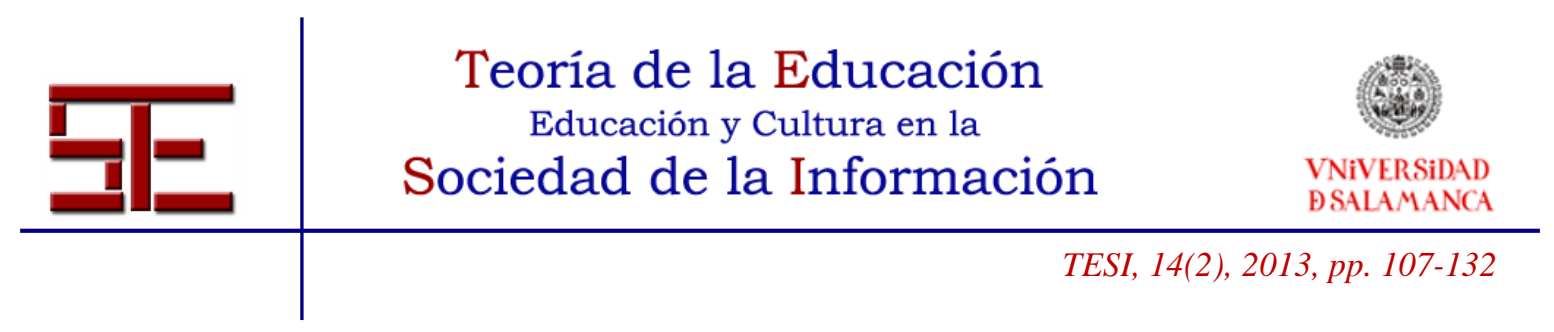

Como se muestra en la Figura 4 el parque está compuesto, además, por plantas, animales y elementos decorativos, entre otros elementos conceptuales. Cada uno de estos conceptos tendrá un desarrollo especial dentro del planteamiento general, que incluirá fotografías, vídeos, textos, etc. Los alumnos/as trabajaron en la búsqueda y selección de información sobre plantas y animales y en la realización de MMCC sobre los elementos decorativos del Parque de la Taconera y su evolución histórica. El ingente trabajo desarrollado se concretó en más de un centenar de MMCC realizados y en un número parecido de fotografías digitales obtenidas, algunos vídeos, y todo ello durante más de una docena de visitas realizadas al parque.

Un modelo de conocimiento se construye sobre el fundamento de un mapa básico y en relación con un tema específico. Consiste en una colección de MMCC vinculados al mapa básico y que representan niveles de diferenciación cada vez más específicos. A los conceptos de los mapas, donde proceda, se vinculan recursos asociados (fotos, documentos, vídeos, etc.), que se designan genéricamente con iconos gráficos. Los MMCC subordinados permiten al usuario navegar a través de ese modelo.

El proceso de construcción de un Modelo de Conocimiento sobre el Parque de la Taconera involucra por lo tanto la elaboración de MMCC que representen el saber de expertos sobre los diferentes aspectos del parque. La elaboración de estos mapas se puede lograr mediante la entrevista a expertos por parte de ingenieros de conocimiento, o pueden ser construidos directamente por los expertos. De cualquier forma, la construcción de la colección de mapas es un proceso iterativo y minucioso para asegurarse la cobertura del tema en los mapas y la fiel representación del conocimiento de los expertos (Coffey, Hoffman, Cañas y Ford, 2002; Crandall, Klein y Hoffman, 2006; Ericsson, Charness, Feltovich y Hoffman, 2006; González y Zuasti, 2008).

Una vez construidos los MMCC, se requiere seleccionar los recursos (fotos, documentos, vídeos, audios, páginas Web, etc.) que mejor complementen y expliquen los conceptos claves en los MMCC. El proceso de recolección de los recursos se puede hacer al mismo tiempo que el desarrollo de los mapas, pero por lo general es mejor ligar los recursos a los mapas cuando los mapas estén revisados y completos. La colección de recursos también sirve para revisar si hay aspectos importantes del parque que no fueron incluidos en los mapas, al encontrarse recursos que no corresponden a ninguno de los mapas.

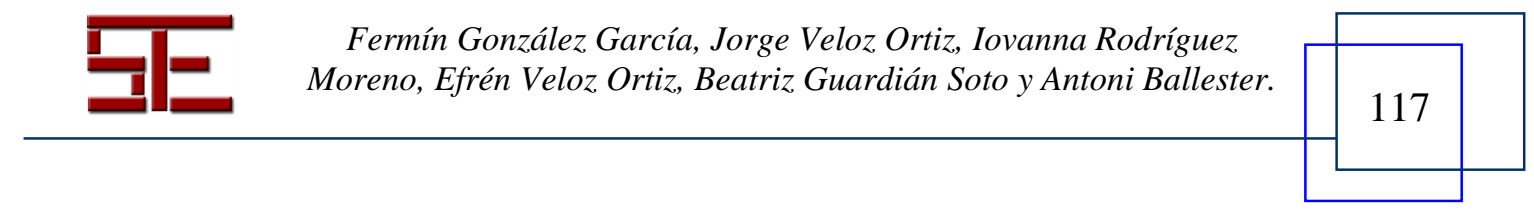




Teoria de la Educación
Educación y Cultura en la
$\begin{gathered}\text { VNiVERSIDAD } \\ \text { BSALAMANCA }\end{gathered}$

La construcción del Modelo de Conocimiento se lleva a cabo utilizando el software CMapTools. Este programa permite enlazar los mapas y recursos creando un sistema hipermedia de fácil navegación para los estudiantes.

En nuestro caso se realizaron MMCC sobre los animales del parque (ver Figura 5) y mamíferos (ver Figura 6). De ellos se obtuvo primero información general y después especializada, referente a cada animal en concreto. Para la organización de los contenidos de los MMCC se realizaron "mapas base", con los que se consiguió mantener unidad y coherencia entre los distintos mapas de un mismo tema. Consecuentemente los mapas que describen las distintas especies reflejaron un arquetipo determinado, posibilitándose así una mejor visibilidad y comparabilidad. Las noticias aparecidas en prensa o Internet relacionadas con el Parque de la Taconera y/o sus componentes fueron también recogidas para ser incluidas posteriormente como material complementario y ser vinculadas a conceptos de los MMCC respectivos.

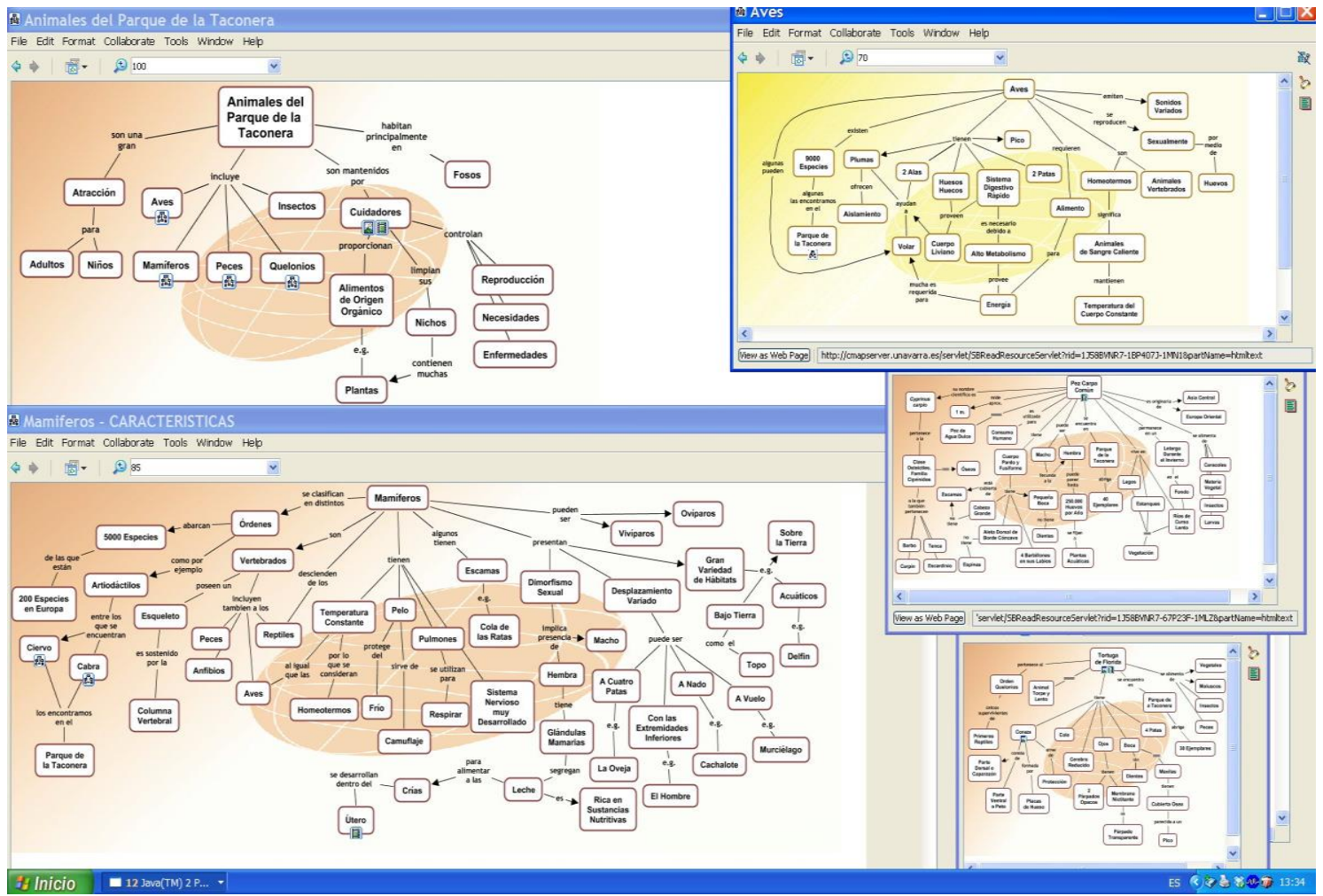

Figura 5. Mapa conceptual de los animales de la Taconera.

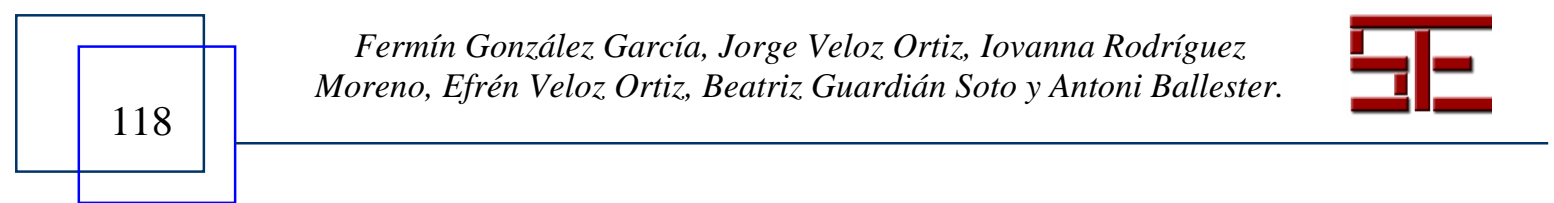




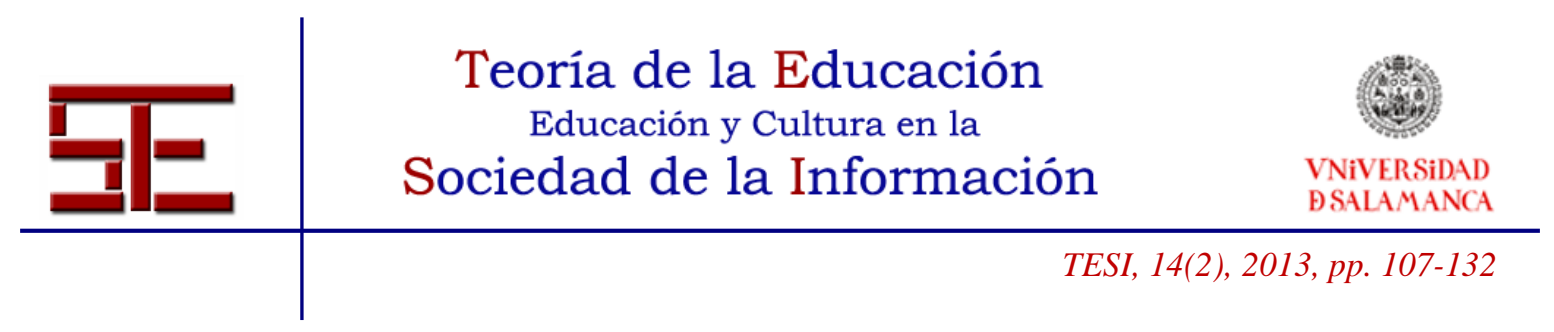

En la figura 5 se muestra el mapa conceptual de los animales de la Taconera así como mapas subordinados describiendo los distintos tipos. Obsérvese cómo podemos navegar a través del modelo de conocimiento construido sobre el Parque de la Taconera utilizando el mapa conceptual como interfaz.

Para ampliar y apoyar la información escrita, se tomaron fotografías de los animales. Se dispuso así de suficientes recursos gráficos para vincular a los conceptos apropiados de los diferentes MMCC. Así pues, para poder sacar las fotos que el Proyecto requería se realizaron numerosas visitas al Parque de la Taconera. Lo que también se abordó fue la realización de un vídeo, que necesitó permiso del Ayuntamiento. Una vez cumplimentada la petición formal previa correspondiente, se filmaron escenas, sobre todo de animales en interiores, y de procesos como incubación de huevos, alimentación de crías, etc. Se rodaron, también, imágenes exteriores, de animales en acción in situ en el interior de los fosos y, asimismo, se sacaron fotografías en recintos reservados del parque y de interés para el proyecto.

El vídeo fue, posteriormente y en pequeños cortes, en un $\mathrm{CD}$, incluido en la página Web, la cual ya en Internet nos permitió dar a conocer nuestro proyecto a todo el mundo. A ella se puede acceder desde la Web principal de la Universidad Pública de Navarra (www.unavarra.es) y a través del capítulo de Investigación, Catálogos de Investigación, Ciencias Humanas y Sociales, Grupo Interdisciplinar de Didáctica de las Ciencias, página Web personal del profesor Fermín González, y, finalmente, Proyecto GONCA.

Queremos significar para finalizar este apartado que esta $4^{\circ}$ fase del proyecto la iniciamos mucho antes que el periodo asignado a la misma en la programación inicial. Esta decisión acertada la tomamos en base a una dificultad prevista y que intuimos, al considerar la complejidad que encerraba la tarea, las condiciones climatológicas, el elevado número de visitas requeridas al Parque de la Taconera para hacer grabaciones de vídeo (que no eran posibles sin la tramitación del permiso oficial correspondiente, al que hemos aludido y que hubo que gestionar), sacar fotografías, etc., de los distintos componentes estructurales del mismo y de sus animales y plantas. Tuvimos siempre muy claro que estos mapas deberían no sólo transparentar la realidad distinta del parque sino, además, la evolutiva y cambiante.

La idea original era que una vez editado el CD con los distintos modelos de conocimiento pudiera ser utilizado en los diferentes niveles educativos navarros y donde procediera.

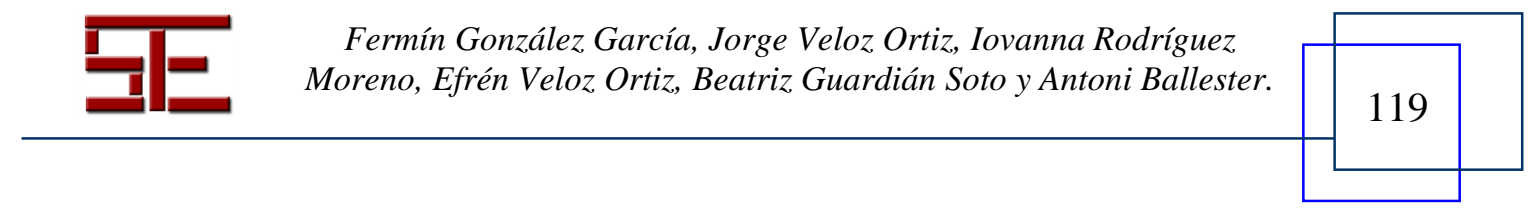



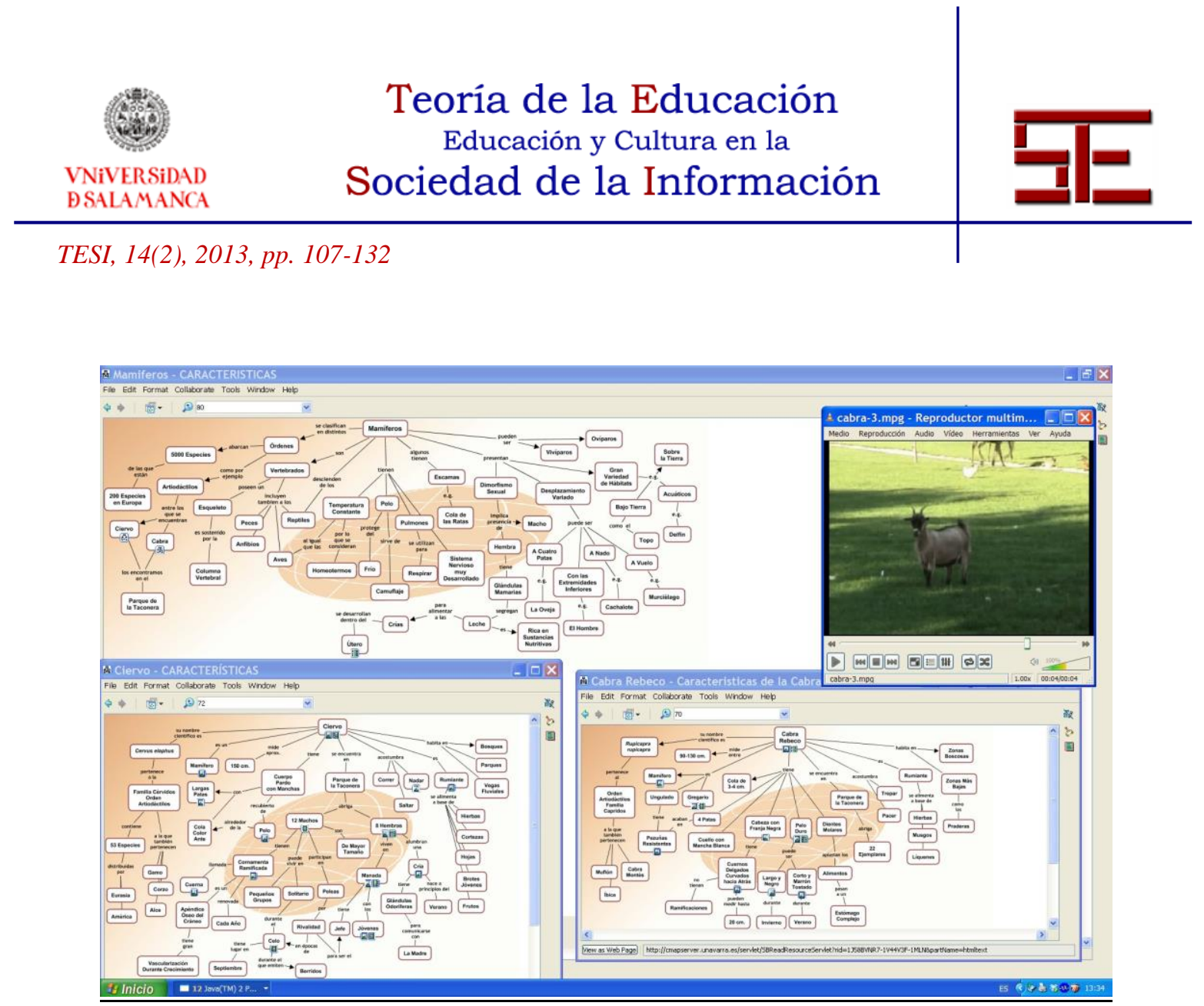

Figura 6. Presenta el mapa conceptual sobre mamíferos con varios recursos desplegados, corresponden a los MMCC del ciervo y la cabra, así como a una foto de ésta última.

Finalmente la Figura 7, muestra el mapa conceptual correspondiente al ciervo y recursos abiertos como fotografías de huellas de dentelladas en su cuerpo, hembra amamantando a su cría, detalle de cuerna vieja, pareja en celo y finalmente cornamenta joven.

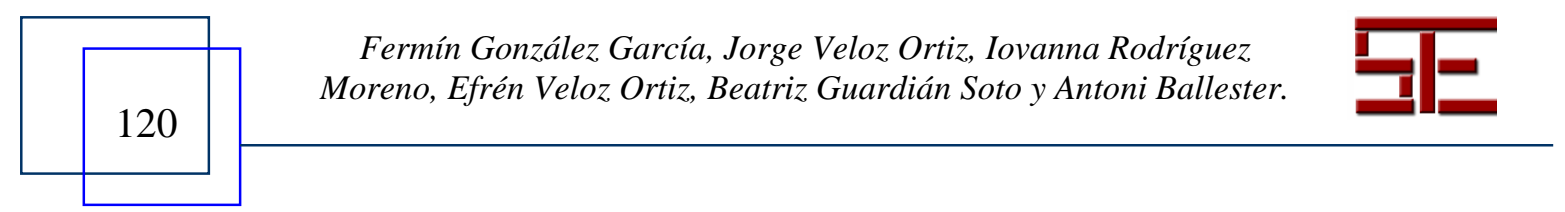



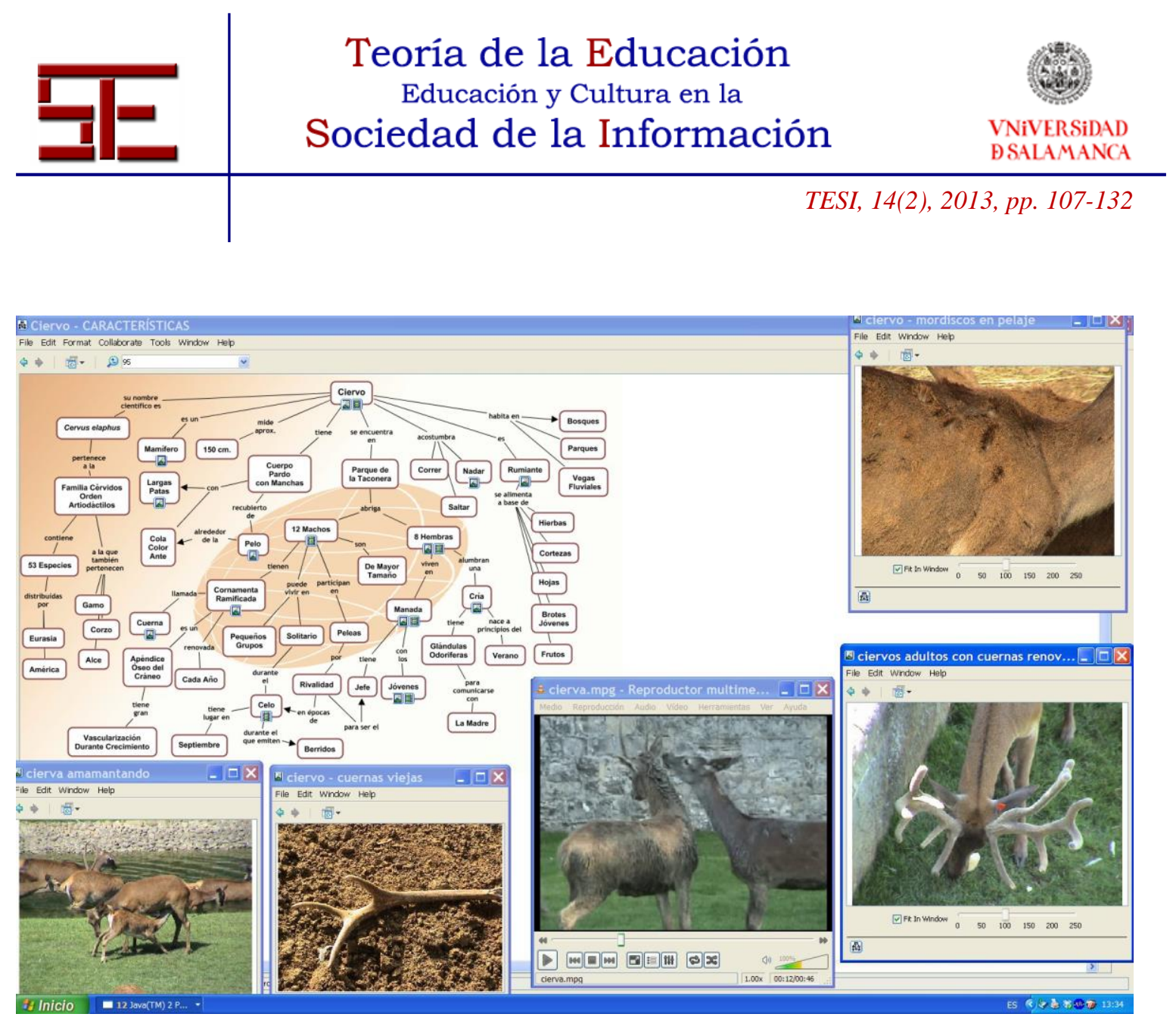

Figura 7. Mapa conceptual sobre el ciervo con algunos recursos desplegados

A modo de resumen y repaso podemos decir que a partir de la Figura 4 que representa el modelo de conocimiento del Parque de la Taconera y los conceptos más generales e inclusivos y utilizando el mapa como interfaz hemos ido navegando haciendo clic en el concepto animales y obtenido el mapa correspondiente a los mamíferos de la Figura 5, el salto representa un grado de especificación mayor de los significados, de mayor detalle. La Figura 6 representa el mapa conceptual de mamíferos y a su vez dos mapas obtenidos al hacer clic respectivamente en los dos ejemplos, ciervo y cabra. En el mapa de esta última se ha desplegado una foto. Finalmente en la Figura 7 se presenta el mapa de detalle del ciervo. Obtenido al hacer clic en el concepto correspondiente de la Figura 6. Se observan además una serie de detalles obtenidos al hacer clic en los correspondientes iconos genéricos gráficos vinculados a los conceptos.

En definitiva, hemos recorrido a través de pisos cada vez más precisos y concretos un edificio/modelo de conocimiento creado en base a informaciones que el alumno ha transformado, previa valoración e incorporación sustantiva a estructuras cognitivas

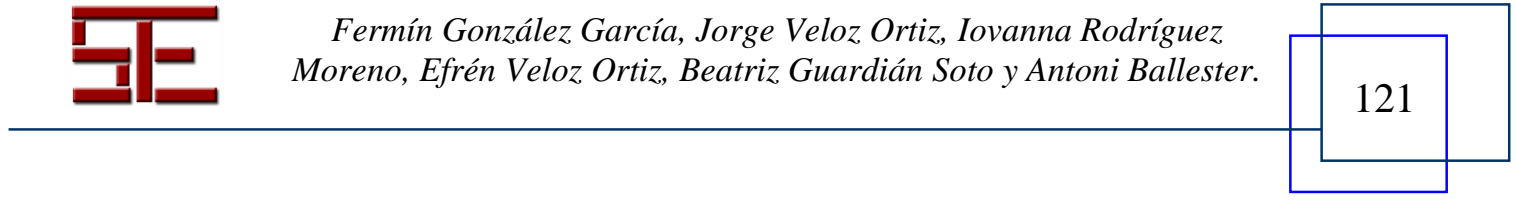




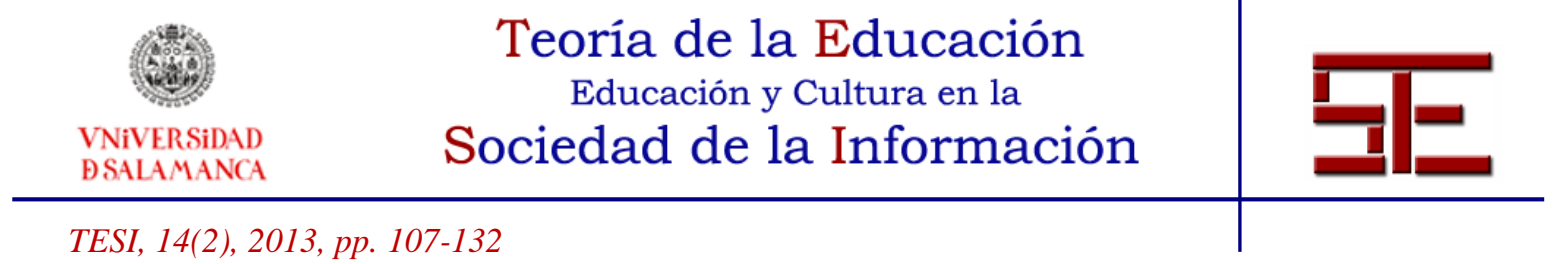

previas, en conocimiento útil. Obsérvese que cada mapa se deriva del anterior, que potencia sus significados. El edificio se ha construido en base a un aprendizaje significativo y es original, pues aunque las informaciones estaban, la reorganización de las mismas, y la re-conceptualización consecuente, ha conducido a la elaboración de un modelo, original y abierto a eventuales mejoras, por unos alumnos/as capacitados/as para añadir valor al mismo con nueva información que será ya relevante para ellos, al tener la mente organizada.

\section{3.- ADAPTACIÓN DE LA ASIGNATURA DIDÁCTICA DEL MEDIO NATURAL AL EEES}

En este contexto del EEES, la organización de la asignatura es fundamental y debe incluir la definición de: objetivos, competencias a desarrollar, programa, metodología, evaluación, planificación temporal y bibliografía. Los cambios más importantes en el EEES van a afectar a aspectos relativos a la metodología y a las competencias. ¿Qué aspectos son clave en la organización de mi asignatura para la convergencia europea? En primer lugar la definición de objetivos en concordancia con los de la titulación. En segundo lugar la definición de las competencias que deben desarrollar los estudiantes. Además tendré que tener en cuenta que el estudiante debe ser el auténtico protagonista del proceso (aprendizaje autónomo). El profesor es el gestor del proceso de aprendizaje, creando las condiciones que favorezcan aquel.

Procede ahora a preguntarse: ¿Cómo incorporo el desarrollo de las competencias a mi asignatura? En primer lugar identificando qué competencias deben desarrollarse en la titulación (el proyecto Tuning tiene una exhaustiva clasificación al respecto, los Libros Blancos de las titulaciones en España -ver Web de la ANECA [Agencia Nacional de la Evaluación de la Calidad y Acreditación]- representan, también, valiosas obras de consulta). En segundo lugar correspondería definir cuáles competencias corresponde potenciar en la asignatura y, finalmente, en tercer lugar habría que fijar la utilización de métodos de enseñanza-aprendizaje que permitan desarrollar simultáneamente los conocimientos teóricos y las habilidades propias de la asignatura.

El aprendizaje autónomo, que adquiere en este contexto una importancia capital, es una forma de aprendizaje en la que el estudiante asume una parte importante de la responsabilidad de la organización de su trabajo, ajustándola a su propio ritmo. ¿Cómo puedo favorecerlo? Motivando a los estudiantes, estableciendo un plan global de trabajo claro, especificando las condiciones del curso, los niveles de exigencia y el sistema de

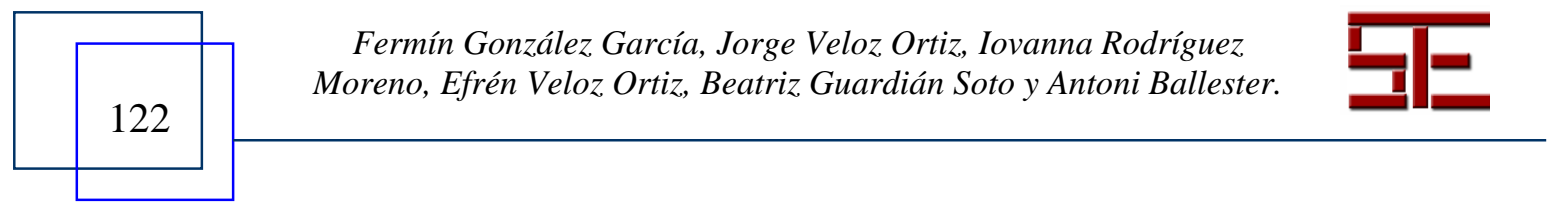




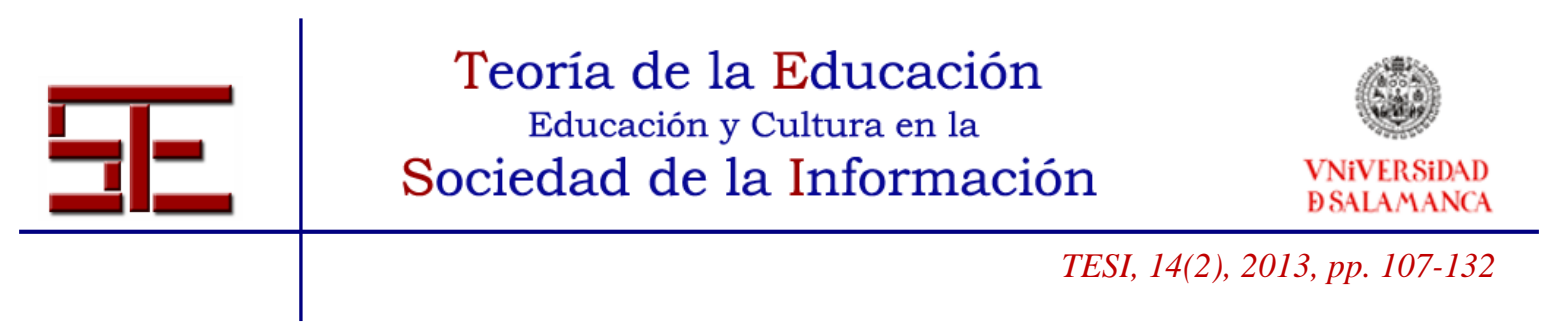

evaluación, disponiendo de un sistema intenso de tutorías y, finalmente, elaborando y entregando un texto-guía a los estudiantes.

La formación a lo largo de toda la vida (Lifelong Learning) es otro de los principios clave de esta nueva cosmovisión que representa el EEES. Según el mismo la universidad no es más que el principio de un proceso formativo y esto debe ser tenido en cuenta al planificar la asignatura. En consecuencia, deberá centrarse en los aspectos fundamentales (aquellos que mejor preparen para la formación continuada), distribuir los contenidos en las diversas fases del proyecto formativo (grado, postgrado, doctorado, formación continua) $\mathrm{y}$, finalmente, crear recursos y dispositivos para la formación continuada. ¿Qué material didáctico puede ayudar en este cambio? Un texto guía, un programa más desarrollado, más "guiador", que propicie el aprendizaje autónomo.

Presentamos, a continuación, la programación de la asignatura Didáctica del Medio Natural adaptada a los presupuestos del EEES y presentando cómo articular la elaboración de modelos de conocimiento en la misma.

\subsection{Mi501. Didáctica del medio natural}

La asignatura se imparte en el $5^{\circ}$ semestre en el Grado de Maestro en Educación Infantil. Competencias generales:

G01, G02, G03, G04, G10, G14, G16, G17, G20, G21, G22, G23, G24, G25, G27, G28, $\mathrm{G} 28$. (Corresponden a las competencias generales especificadas con esa denominación por la ANECA).

Competencias específicas:

1.- Conocer los fundamentos científicos y tecnológicos del currículo de esta etapa así como las teorías sobre la adquisición y desarrollo de los aprendizajes correspondientes.

2.- Conocer la metodología científica y promover el pensamiento científico y la experimentación.

3.- Conocer los momentos más sobresalientes de la historia de las ciencias naturales y las técnicas y su trascendencia.

4.- Promover el interés y el respeto por el medio natural a través de proyectos didácticos adecuados.

5.- Fomentar experiencias de iniciación a las tecnologías de la información y la comunicación.

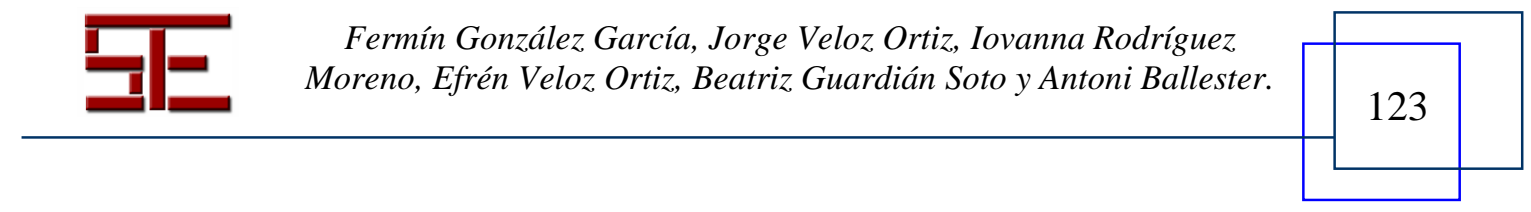




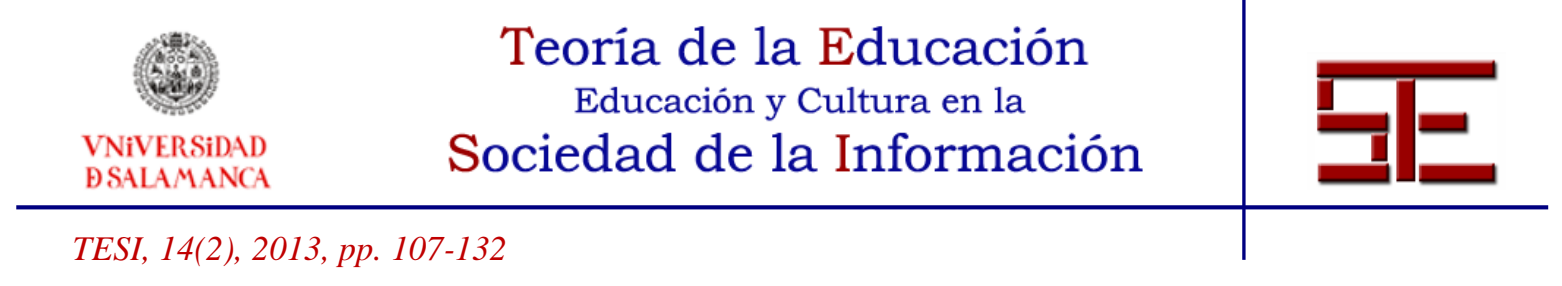

6.- Ser capaz de elaborar proyectos didácticos sobre el medio natural, utilizando situaciones didácticas que fomenten el aprendizaje constructivo y promuevan el interés y el respeto por el medio natural.

7.- Ser capaz de exponer a los alumnos a sencillos problemas científicos de modo que desarrollen una actitud positiva respecto a la actividad indagadora y científica, practiquen el trabajo autónomo así como la construcción social del conocimiento a través del trabajo en equipo.

8.- Programar y desarrollar sencillos proyectos científicos que permitan la puesta en práctica de procesos básicos de la actividad científica: coleccionar, observar, estimar, medir, clasificar, ordenar, organizar datos, inferir reglas, predecir.

\subsubsection{Descriptores}

Teoría del aprendizaje significativo para el conocimiento del medio natural. Fomento de actitudes positivas y conductas coherentes de respeto al medio natural. El entorno próximo como recurso didáctico fundamental. Procedimientos y recursos para la enseñanza de la Didáctica del medio natural.

\subsubsection{Metodología}

Básicamente está diseñada para que el alumno/a pase por las fases imprescindibles para obtener un auténtico dominio del conocimiento, esto es: Adquisición de la información (a través de clases magistrales y estudio de documentos pertinentes), consolidación de la información que pasa ya a conocimiento útil (a través del trabajo de seminario con interacción constante con el profesor de la asignatura y la elaboración subsiguiente de los modelos creativos de conocimiento sobre los temas elegidos) y, finalmente, la fase de asesor/diseminador del conocimiento construido, a través de la presentación pública del modelo de conocimiento construido por el grupo.

Tanto la teoría como la práctica, que está informada en todo momento por la teoría del aprendizaje significativo, se impartirán, siempre que sea posible, simultáneamente, con la ayuda del trabajo en los seminarios. La metodología específica práctica utilizada en el trabajo en pequeños grupos incluye actividad grupal y trabajo autónomo, incluyendo las siguientes fases:

1.- Presentación del trabajo a realizar y sensibilización.

2.- Entrega de material y referencias bibliográficas y recursos de Internet, constitución de los grupos de trabajo y nombramiento de responsables de grupo.

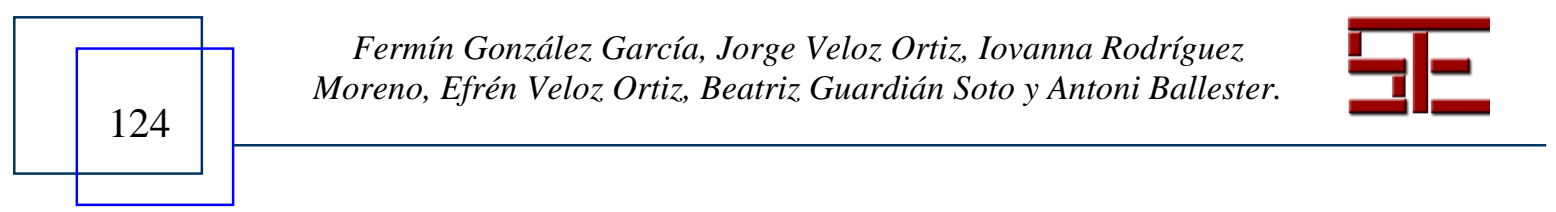




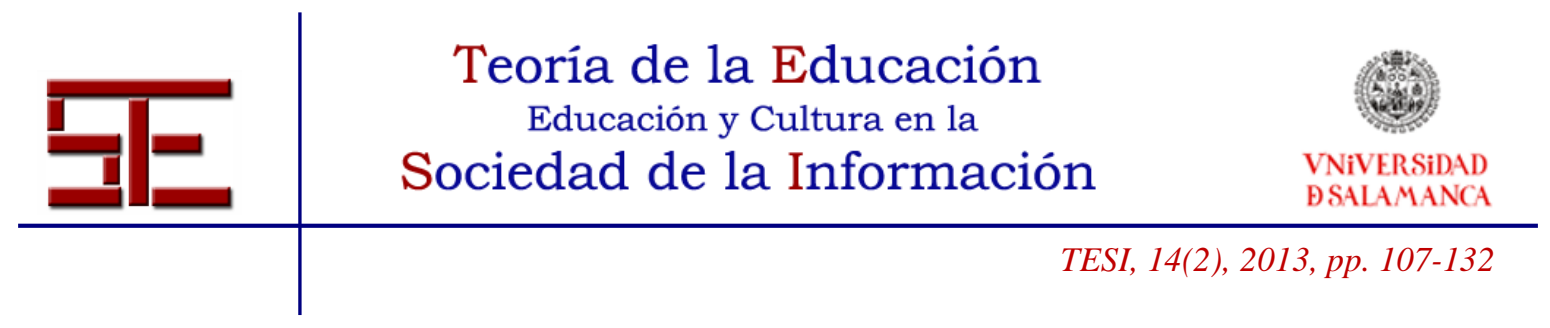

3.- Elaboración del trabajo en clase bajo la supervisión del profesor. Esta fase incluye los procesos siguientes:

- Reflexión y planteamiento teórico del trabajo a partir del diagrama V de Gowin.

- Búsqueda y organización de la información.

- Elaboración de un módulo instruccional sobre un tema del entorno próximo (Campus UPNA, Cuenca de Pamplona, Recursos didáctico-geológicos de Pamplona y sus alrededores).

4.- Exposición de los trabajos utilizando las nuevas tecnologías (TIC) y el programa Cmap Tools.

\subsubsection{Actividades formativas}

- Clase magistral (grupo general) para introducción de los conceptos relevantes del temario.

- Participación activa del alumnado en clase/seminario/tutorías/prácticas de campo y en situaciones: individual, pequeño grupo, gran grupo, para conocer el contexto real de sus conocimientos, propiciando el profesor una atmósfera familiar, que estimule las intervenciones orales del alumnado.

- Excursiones (si es posible), paseos botánicos por el campus de la UPNA, paseos urbanos.

- Elaboración de un herbario, a cargo de los grupos concernidos.

- Elaboración de una maqueta de la cuenca de Pamplona, por los grupos involucrados.

- Estudio autónomo, personal y en pequeño grupo.

- Seminarios de dudas y de seguimiento del proceso de elaboración del modelo de conocimiento, para discutir, aclarar y compartir, finalmente, los conocimientos.

- Las competencias básicas científicas, fundamentalmente, observación, registros sistemáticos y experimentación se desarrollarán mayoritariamente en la naturaleza, en las prácticas de campo.

- El dominio de las TIC se conseguirá a través de los ordenadores personales que se traerán, en todo momento, a las clases y seminarios y mediante la familiarización con el Cmap Tools software.

- Presentación pública de los modelos creativos de conocimiento construidos por los distinto grupos, y que están elaborados para ser llevadas al aula de Educación Infantil.

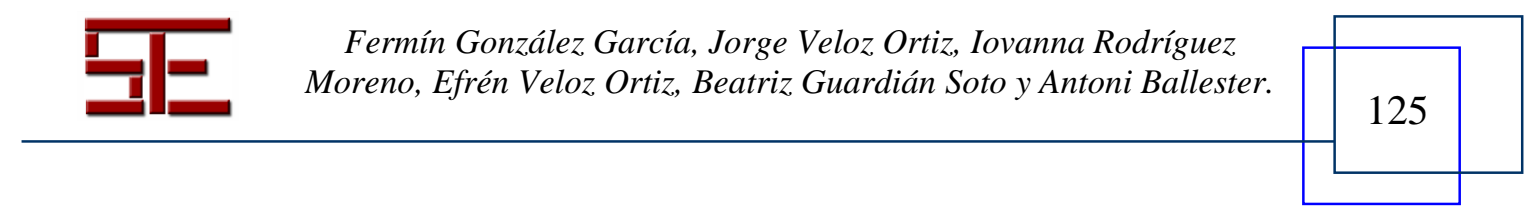




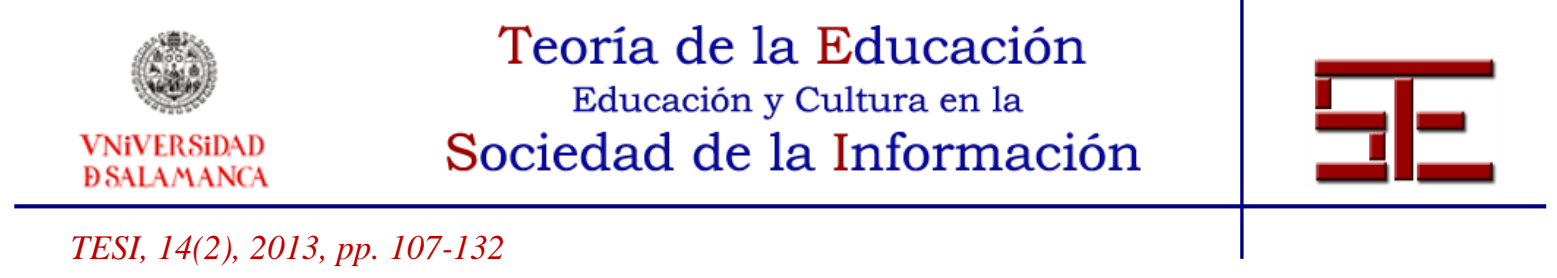

Idiomas: Castellano.

\subsubsection{Contenidos}

El temario está estructurado teniendo en cuenta la psicología del aprendizaje (cognitivo/constructivista) subyacente al planteamiento de la asignatura. Con un Bloque I diseñado para sentar las bases teóricas que permitan al alumno aprender significativamente y construir conocimientos (a través de los procesos de investigación correspondientes) y para dotarles de los instrumentos apropiados para llevar a cabo con éxito estas tareas. Así mismo se pretende que el alumnado conozca el currículo de Educación infantil, en lo relativo al Conocimiento del, entorno que se aplica en la Comunidad Foral de Navarra.

En el Bloque II se aplican todos los conocimientos teóricos y teórico-prácticos adquiridos en el Bloque I a la elaboración de un modelo de conocimiento sobre temas determinados del medio natural, que evidencien el conocimiento adquirido por los alumnos y su dominio, a través de la presentación pública del modelo.

\subsubsection{BLOQUE I}

Tema 1: Análisis de los modelos educativos: tradicional (conductista y positivista) y progresista (cognitivo y constructivista).

Teorías de la enseñanza/aprendizaje de Ausubel, Novak y Gowin, para la Didáctica del medio natural.

Principios fundamentales de las mismas.

Teoría de los errores conceptuales.

Tema 2: Técnicas instruccionales para la mejora de los procesos de enseñanza/aprendizaje del medio natural.

2.1. Los MMCC y el aprendizaje significativo del medio natural.

2.2. El diagrama $\mathrm{V}$ del conocimiento, el aprendizaje significativo del medio natural, la construcción de conocimientos y el proceso de investigación del medio natural.

Tema 3: Currículo de Educación Infantil en la Comunidad Foral de Navarra.

3.1. Conocimiento del entorno: objetivos, contenidos y criterios de evaluación.

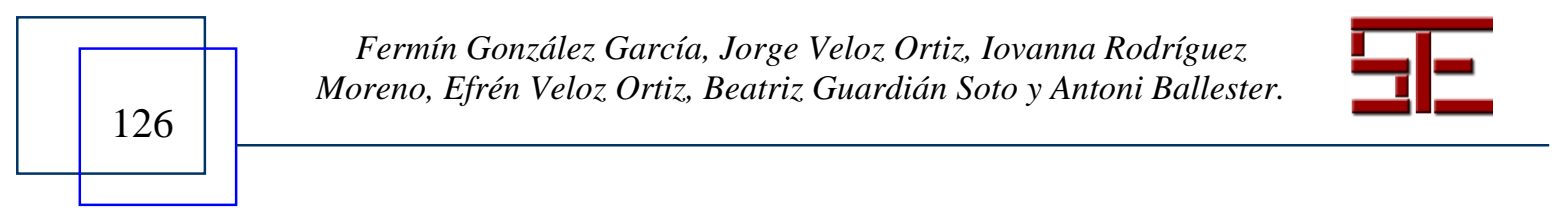




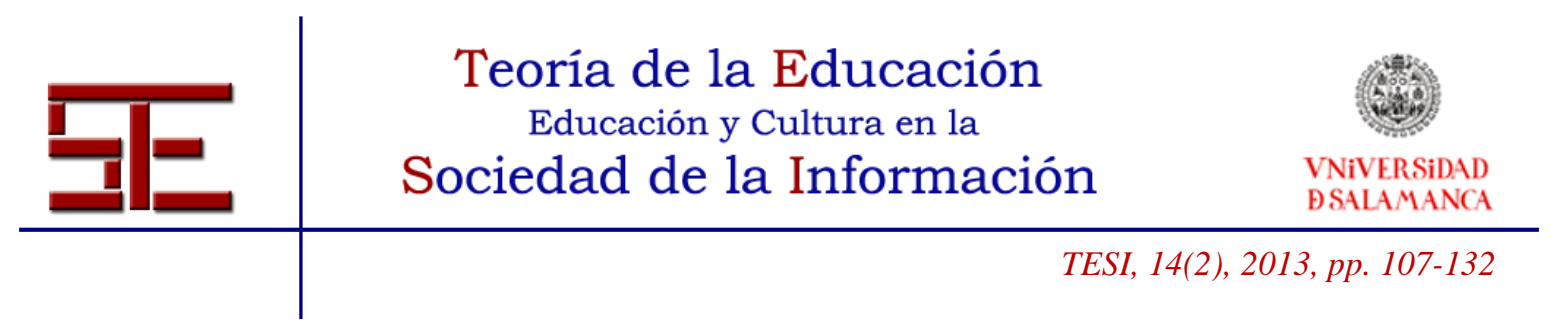

Los temas del Bloque I está enfocados para la obtención fundamentalmente de las competencias específicas: $1,2,4,5$,

\subsubsection{BLOQUE II}

Tema 4: Elaboración de un diseño curricular e instruccional en relación con aspectos seleccionados del medio natural (Campus UPNA, Cuenca de Pamplona, Recursos didáctico-geológicos de Pamplona y sus alrededores).

4.1. Pautas para la elaboración de un diseño curricular e instruccional en relación con el medio natural.

4.2. Elaboración del modelo de conocimiento correspondiente.

4.3. Presentación pública del mismo.

Los temas del Bloque II están enfocados para la obtención fundamentalmente de las competencias específicas $2,4,5,6,7$ y 8 .

\subsubsection{Evaluación}

La evaluación consistirá en una prueba escrita y en la elaboración de un módulo instruccional sobre el área de Conocimiento del medio.

En ambos casos se tratará de verificar si los estudiantes han conseguido las competencias propuestas para esta asignatura.

En la prueba escrita, que supondrá el $50 \%$ de la calificación final, tendrán que dar respuesta a una serie de cuestiones teóricas y prácticas referentes al programa explicado por el profesor y a las prácticas realizadas en clase.

La valoración del módulo instruccional realizado y su presentación oral supondrá el 50\% restante.

\subsubsection{Temario (ver contenidos)}

\subsubsection{Bibliografía}

ACTAS de los Congresos que sobre aplicación de los MMCC para la mejora de los procesos de enseñanza/aprendizaje se celebraron en Pamplona (2004), San José de Costa Rica (2006), Helsinky (Finlandia) y Tallin (Estonia) en 2008, y en Viña del Mar (Chile, 2010) ( descarga libre en http://cmc.ihmc.us).

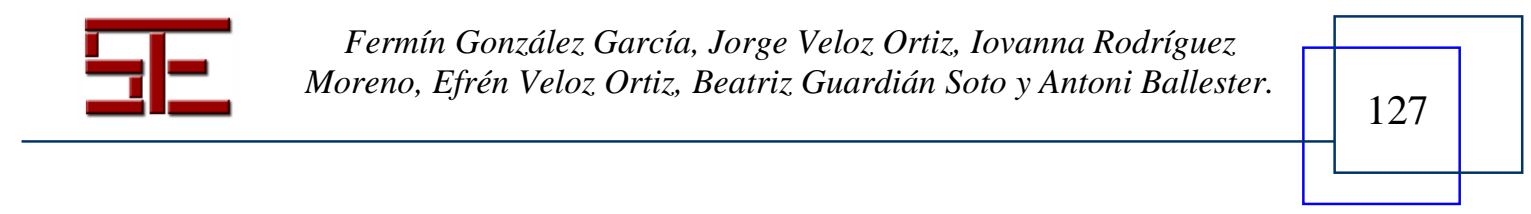




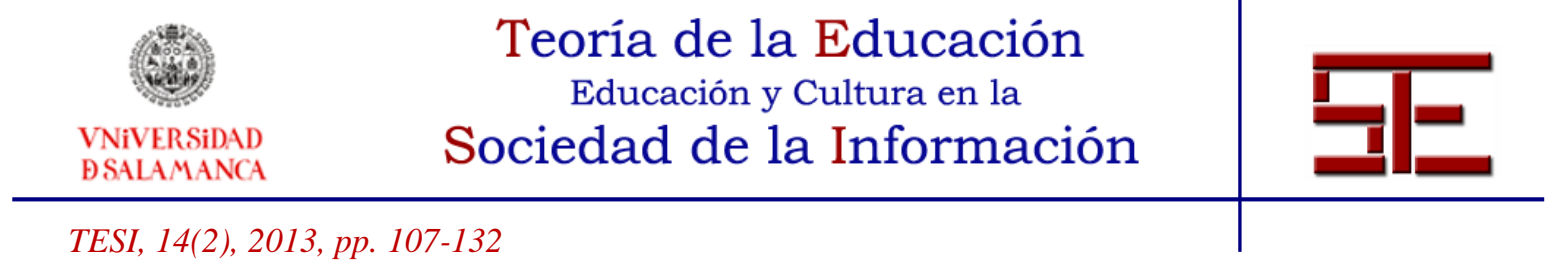

Documentos oficiales sobre el currículo de Educación Infantil en la Comunidad Foral de Navarra. Gobierno de Navarra. Departamento de Educación.

González, F. (2008, 2ª edición). El mapa conceptual y el Diagrama UVE. Recursos para la Enseñanza Superior en el siglo XXI. Madrid: Narcea.

González, F. Ibáñez, F. Casalí, J., López, J. y Novak J. D. (2007). Una aportación a la mejora de la calidad de la docencia universitaria: Los MMCC. Pamplona: Servicio de Publicaciones de la Universidad Pública de Navarra.

González, F. e Iza, J. Mª (1986). Recursos didáctico-geológicos de Pamplona y sus alrededores. Pamplona: Ayuntamiento de Pamplona.

NOTA. Se proporcionará bibliografía especializada y adecuadas orientaciones a los distintos grupos en función de los temas elegidos. Así mismo se informará, en todo momento, sobre bibliografía personalizada y otros recursos a demanda.

Toda la información para profundizar en estos aspectos, tanto teóricos como prácticos, y para la eventual mejora de la gestión de la asignatura se puede consultar en el servidor público de CmapTools de la Universidad Pública de Navarra. En él, la carpeta titulada Fermín González- Univ. Pública Navarra-España y, finalmente, la subcarpeta DIDÁCTICA DEL MEDIO NATURAL.

\section{4.- REFLEXIONES FINALES}

Hemos tratado en este artículo de la importancia de considerar el EEES y las connotaciones de cambio del paradigma educativo que implica. La vocación de liderazgo mundial con que irrumpe el nuevo modelo y las prometedoras respuestas que propone para los desafíos que se plantean desde la llamada Sociedad del Conocimiento y de la Información y desde la aplicación de los Paradigmas de Calidad para una mejora continua en los procesos (González, 2008) son atractivos suficientes para considerar el cambio que propone, desde otras realidades.

En este artículo hemos enfatizado también la utilidad del marco teórico/práctico de Ausubel, Novak y Gowin, así como la de los MMCC, ahora multiplicado su potencial por el extraordinario software CmapTools (Cañas, 2004), integrados totalmente en nuestro quehacer profesional diario, para la mejora de la calidad de la docencia. Este planteamiento constituye la base del diseño e implementación de nuestra asignatura para lograr el aprendizaje significativo de nuestros estudiantes, así como de la construcción creativa de conocimientos a través de la elaboración de modelos de conocimiento

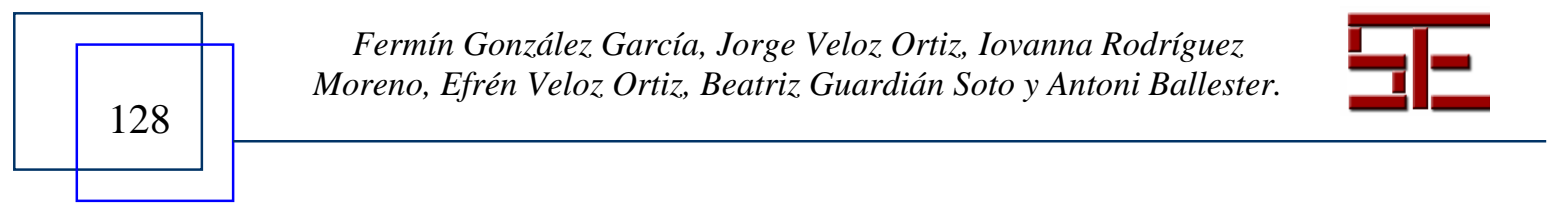




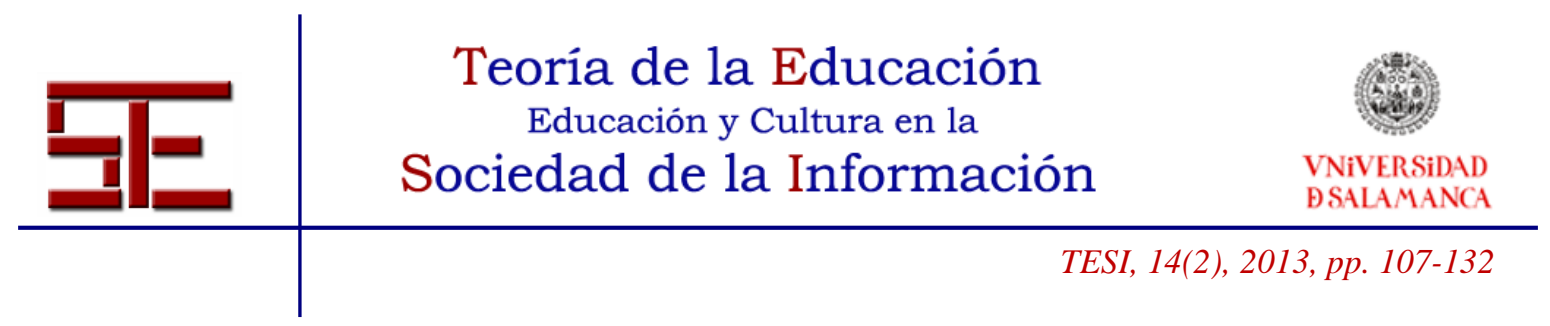

originales. La puesta en práctica de esta estrategia ha facilitado la adaptación de nuestras asignaturas a los requisitos del EEES. Evidencias que soportan estos juicios de valor son los resultados de investigaciones propias (Albisu, San Martín y González, 2006; Ballester, 2008; Guardián, 2009; Guruceaga, 2001; Pozueta, 2011).

Los modelos de conocimiento construidos por los alumnos/as facilitan el aprendizaje significativo y la construcción creativa de conocimientos, a través de un proceso que queda abierto para facilitar el aprendizaje de los alumnos/as y la construcción de conocimientos de por vida.

\section{5.- BIBLIOGRAFÍA}

Albisu, S., San Martin, I. \& González, F. (2006). Aplicación de los Mapas Conceptuales y de la V de Gowin en la Elaboración de Módulos Instruccionales en Alumnos de Magisterio. En Proceedings of the_Second International Conference on Concept Mapping. San José, Costa Rica.

Ballester, A. (2002). El aprendizaje significativo en la práctica. Cómo hacer el aprendizaje significativo en el aula. Libro digital gratuito en www.aprendizajesignificativo.com pp. 192. Traducción al inglés (2011). Meaningful learning in practice. How to put meaningful learning in the classroom. En www.meaningfullearning.com. Traducción al catalán (2011). L'aprenentatge significatiu a la pràctica. Com fer l'aprenentatge significatiu a l'aula. www.aprenentatgesignificatiu.com. Extraído el 15 de noviembre de 2011.

- (2008). Meaningful learning in the practice. En Proceedings of the 3rd International Conference on Concept Mapping. (pp. 185-188). Helsinki (Finlandia) y Tallin (Estonia).

Cañas, A. (2004). Cmaptools: A Knowledge Modeling And Sharing Environment. Proceedings of the First International. Conference on Concept Mapping. (pp. 125-133). Pamplona, Spain.

Coffey, J., Hoffman, R., Cañas, A. \& Ford, K. (2002). A Concept Map - Based Knowledge modeling approach to Expert Knowledge Sharing. IKS. The IASTED International Conference on Information and Knowledge Sharing. November. Virgin Islands.

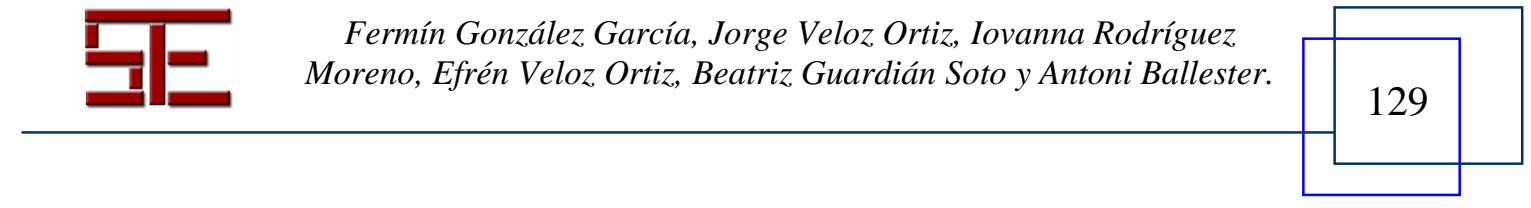




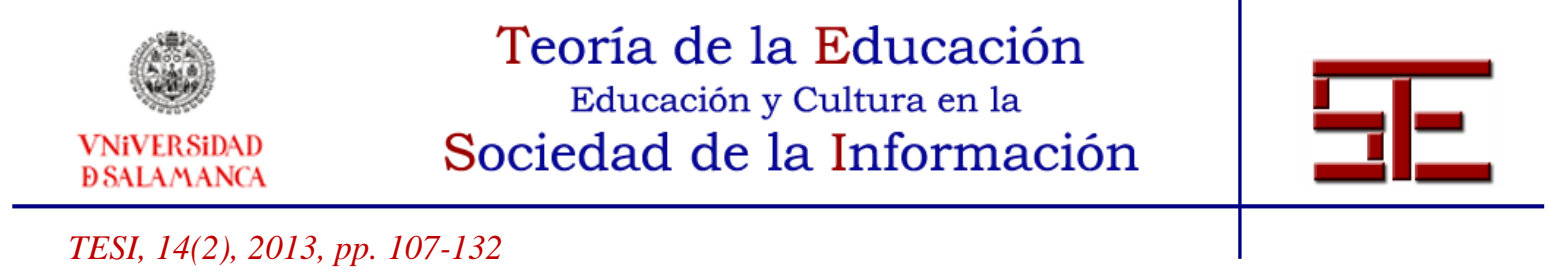

Crandall, B., Klein, G. \& Hoffman, R. (2006). Working Minds. A Practitioner's Guide to Cognitive Task Analysis. MIT Press: Cambridge, MA.

Ericsson, K., Charness, N., Feltovich, P. \& Hoffman, R. (2006). The Cambridge Handbook of Expertise and Expert Performance. Cambridge University Press: Cambridge, MA.

González, F. (2008). El Mapa Conceptual y el Diagrama V. Recursos para la Enseñanza Superior en el Siglo XXI. Madrid: Narcea.

González, F., Guruceaga, A., Pozueta, E. y Lara, R. (2009). Making visible good teaching practices of a university lecturer by using concept mapping. International Association for the Development of Advances in Technology IADAT. 5th IADAT International Conference on Education. Bilbao (Spain), June 24-26.

González, F., Morón, C. \& Novak, J. (2001). Errores conceptuales. Diagnosis, tratamiento y reflexiones. Pamplona: Ediciones Eunate.

González, F. \& Wagenaar, R. (2003). Tuning Educational Structures in Europe. (Informe Final Proyecto Piloto-Fase1). Bilbao: Universidad de Deusto.

González, F. \& Zuasti, J. (2008). The Running of the Bulls. A Practical Use of Concept Mapping to Capture Expert Knowledge. En Proceedings of 3rd International Conference on Concept Mapping. (pp.242-245). Tallin, Helsinki,

Guardián, B. (2009). La Teoría de Ausubel-Novak- Gowin en la Enseñanza del Diseño y Análisis de los Algoritmos Computacionales en el IPN. Tesis Doctoral inédita. Universidad Autónoma Metropolitana de México, Xochimilco.

Guruceaga, A. (2001). Ikaskuntza Esanguratsua eta Ingurugiro Hezkuntza (Aprendizaje Significativo y Educación Ambiental). Tesis Doctoral inédita. Universidad Pública de Navarra.

Meichenbaum, D. \& Biemiller, A. (1998). Nurturing Independent Learners. Helping Students Take Charge of their Learning. Cambridge, Massachusetts: Brookline Books

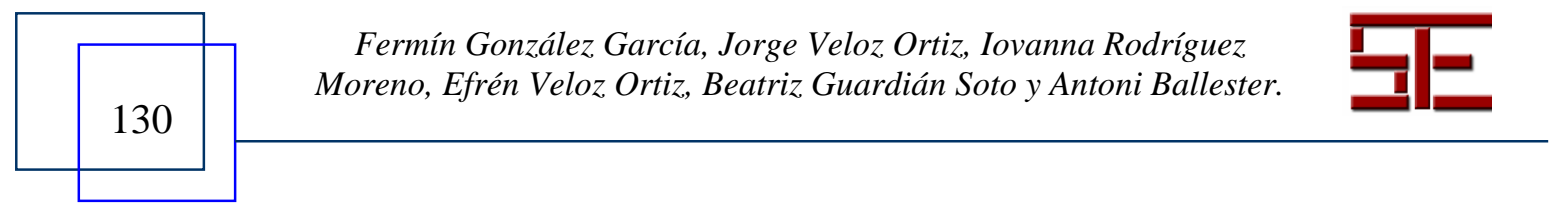




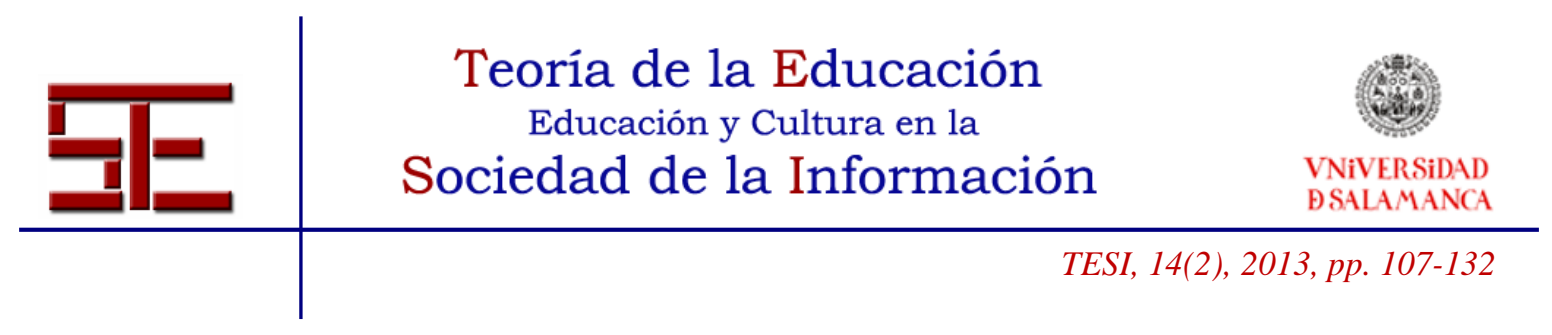

Molina, M.; Pozueta, E. \& González, F. (in progress). Diagnóstico y Tratamiento de Errores Conceptuales. Tesis de Doctorado. Universidad Pública de Navarra.

Novak, J. (1998). The Pursuit of a Dream: Education Can be Improved. En J. Mintzes, J. Wandersee, \& J. Novak. Teaching Science for Understanding. A Human Constructivist View. (pp. 3-28). San Diego: Academic Press.

- (1998). Learning, creating and using knowledge: Concept maps as facilitative tools in schools and corporations. Mahwah, NJ: Lawrence Erlbaum Associates.

Novak, J. \& Cañas, A. (2006). The Theory Underlying Concept Maps and How to Construct Them (Technical Report IHMC CmapTools 2006-01). Florida Institute for Human and Machine Cognition.

Novak, J. \& Gowin, D. B. (1988) Aprendiendo a aprender. Barcelona: Martínez Roca.

Posner, G., Strike, K., Hewson, P. \& Gertzog, W. (1982). Accomodation of a scientific conception: toward a theory of conceptual change. Science Education, 62 vol.2, 211-227.

Pozueta, E. (2011). Una Aplicación del Modelo Cognitivo Constructivista y de los Mapas Conceptuales para la Mejora de la Enseñanza de las Matemáticas en Educación Secundaria Obligatoria. Tesis Doctoral inédita. Universidad Pública de Navarra.

Proyecto GONCA (2003). San Martin, I., Albisu, S. \& González, F. (2008). Constructing Knowledge Models. Cooperative Autonomous Learning Using Concept Maps and V Diagrams. Proceedings of the $3^{\text {rd }}$ International Conference on Concept Mapping. Tallin, Helsinky (pp.140-143).

Extraído http://www.unavarra.es/invest/GONCA/

Villar, L. y Alegre, O. (2004). Manual para la Excelencia en la Enseñanza Superior. Madrid: McGraw Hill.

Zabalza, M. (2004). Guía para la Planificación Didáctica de la Docencia Universitaria en el marco del Espacio Europeo de Educación Superior (Guía de guías). Documento de trabajo.

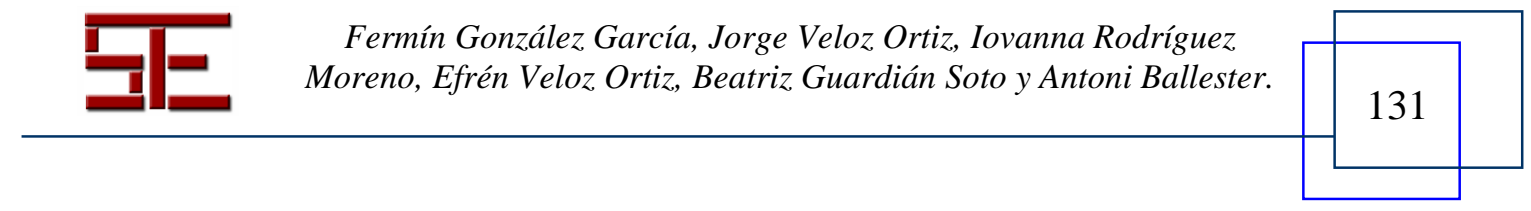




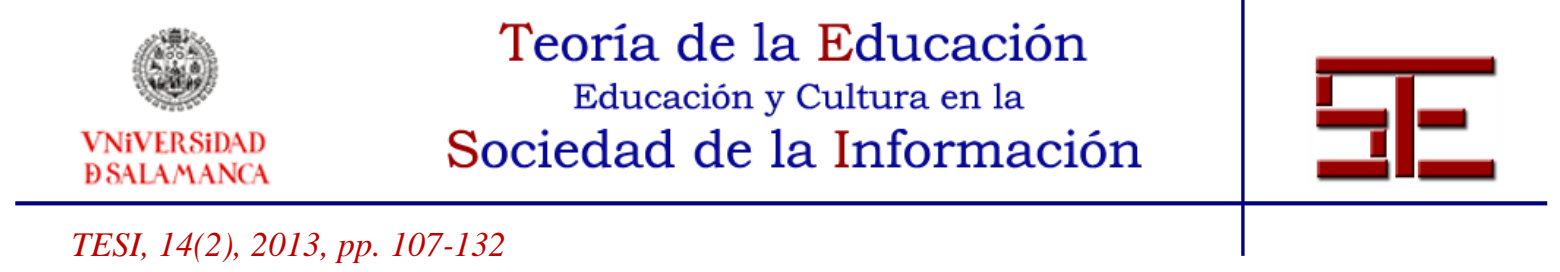

Para citar el presente artículo puede utilizar la siguiente referencia:

González García, F. M., Veloz Ortiz, J. F., Rodríguez Moreno, I. A., Velos Ortiz, L. F., Guardián Soto, B. y Ballester Valori, A. (2013). Los modelos de conocimiento como agentes de aprendizaje significativo y de creación de conocimiento. Revista Teoría de la Educación: Educación y Cultura en la Sociedad de la Información. 14(2), 107-131 [Fecha de consulta: dd/mm/aaaa]

http://campus.usal.es/ revistas_trabajo/index.php/revistatesi/article/view/10216/10625 